LA-UR-04-6852

DOE/NV11718--990

\title{
MEASUREMENT OF THE SHOCK-HEATED MELT CURVE OF LEAD USING PYROMETRY AND REFLECTOMETRY
}

\author{
D. Partouche-Sebban ${ }^{\mathrm{a}}$ and J. L. Pélissier \\ Commissariat à l'Energie Atomique, 91680 Bruyères le Châtel, France
}

F. G. Abeyta, W. W. Anderson, M. E. Byers, D. Dennis-Koller, J. S. Esparza, R. S. Hixson, D. B. Holtkamp, B. J. Jensen, J. C. King, P. A. Rigg, P. Rodriguez, D. L. Shampine, J. B. Stone, and D. T. Westley

Los Alamos National Laboratory, Los Alamos, New Mexico 87545, U.S.A.

\begin{abstract}
S. D. Borror and C. A. Kruschwitz
Bechtel Nevada, Los Alamos Operations, P.O. Box 809, Los Alamos, New Mexico, 87544, U.S.A.
\end{abstract}

\begin{abstract}
Data on the high-pressure melting temperatures of metals is of great interest in several fields of physics including geophysics. Measuring melt curves is difficult but can be performed in static experiments (with laser-heated diamond-anvil cells for instance) or dynamically (i.e., using shock experiments). However, at the present time, both
\end{abstract}

\footnotetext{
${ }^{a}$ Currently at Los Alamos National Laboratory
} 
experimental and theoretical results for the melt curve of lead are at too much variance to be considered definitive. As a result, we decided to perform a series of shock experiments designed to provide a measurement of the melt curve of lead up to about $50 \mathrm{GPa}$ in pressure. At the same time, we developed and fielded a new reflectivity diagnostic, using it to make measurements on tin. The results show that the melt curve of lead is somewhat higher than the one previously obtained with static compression and heating techniques.

PACS: $\quad 62.50 .+\mathrm{p}, 64.70 . \mathrm{Dv}, 8130 \mathrm{Bx}, 8170 \mathrm{Fy}$

\section{INTRODUCTION}

Before the investigation in 1990 of the melt curve of lead (temperature vs. pressure) with a laser-heated diamond-anvil cell up to $100 \mathrm{GPa},{ }^{1}$ static compression methods were limited to about $10 \mathrm{GPa}$ (see Refs. 2 and 3). It has been previously shown in shock experiments that the incipient melting point on the Hugoniot curve was in the vicinity of $50 \mathrm{GPa},{ }^{4}$ a result later confirmed by the observation that sound velocity of lead was that of a liquid above $54.5 \mathrm{GPa}^{5}$ and by a theoretical calculation of the Hugoniot and the melting curve. ${ }^{6}$ However, at high pressures, the melting of lead in Ref. 1 is inconsistent with, and lower than, the prediction of Ref. 6.

As a result, we undertook to measure the melt curve of lead using shock techniques. A previous temperature measurement ${ }^{7}$ near $50 \mathrm{GPa}$ published earlier remains qualitative because a gray-body analysis was used over a large wavelength range. Our approach is to shock the metal near the incipient melting point on the Hugoniot curve (i.e., near $50 \mathrm{GPa}$ in shock pressure ${ }^{4}$ ). In this way, the material is initially fully solid (or 
with a small amount of liquid phase) but the release isentrope largely follows the melting curve and the liquid proportion increases when pressure decreases along the isentrope (see Fig. 1). We used three different windows — sapphire, lithium fluoride (LiF) and polymethylmethacrylate (PMMA). With the window acting as a shock "anvil" preventing full pressure release at the metal/anvil interface, we can measure three points on the metal melt curve with a single initial shock pressure. Note, however, that in the case of PMMA, lead can turn fully liquid on release if the initial shock pressure is high enough (55 GPa, according to our theoretical estimates with the models used in Ref. 6). The experiments produced initial shock pressures of approximately 49,50 and $57 \mathrm{GPa}$ and we obtain several experimental points on the melt curve in the 10 to 50 GPa pressure range by using these several types of windows.

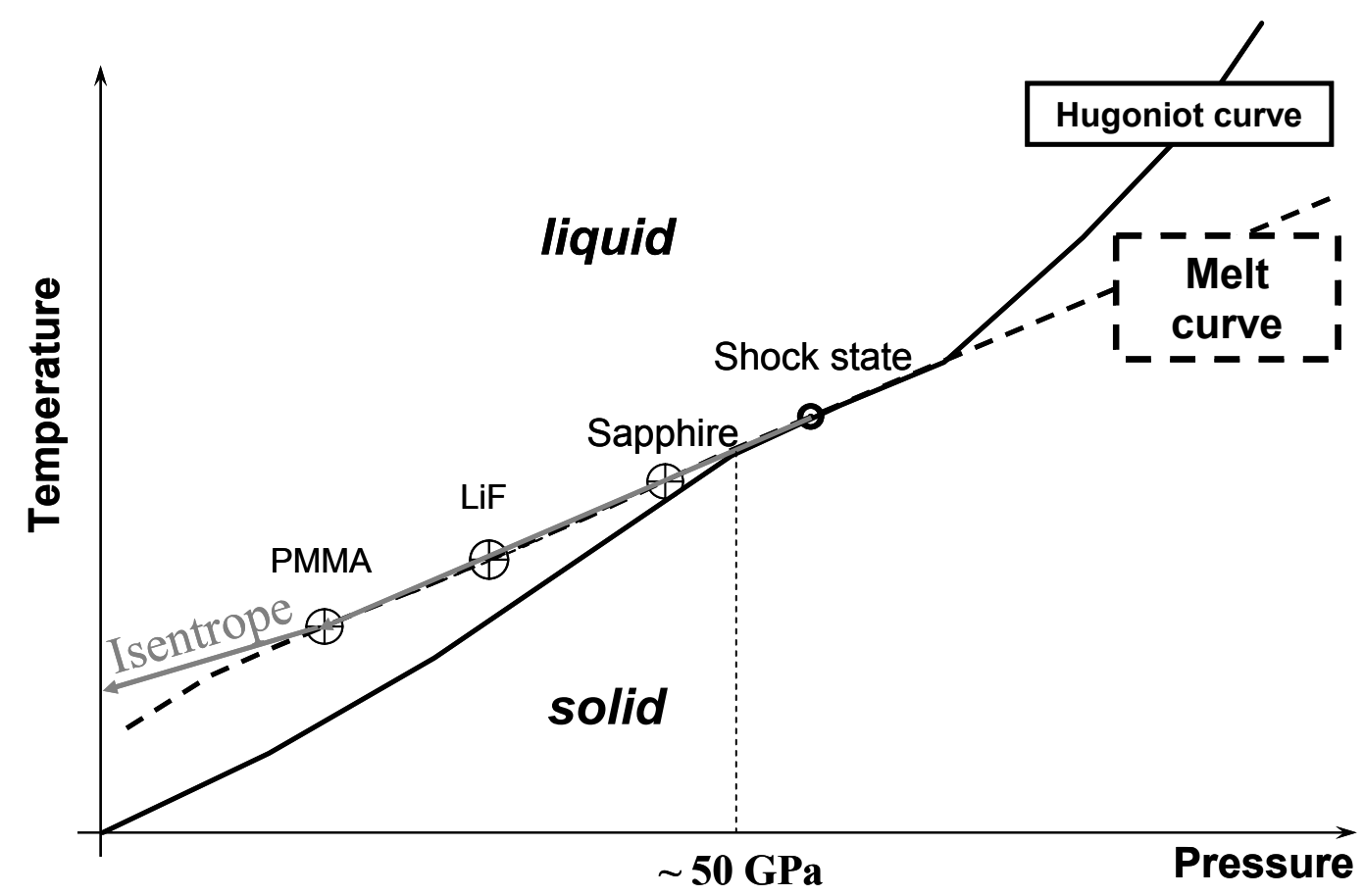

Fig. 1: Principle of the measurement of the melt curve of lead using shock experiments. A transparent anvil (PMMA, LiF or sapphire) is glued onto the lead sample to allow partial release of the metal (down to some intermediate pressure 
determined by the window shock-impedance). From a single shock state near the incipient melting point on the Hugoniot curve, using different anvil materials gives the possibility to measure several points on the melt curve.

Using a similar approach, we performed a measurement on tin with a PMMA window, initially from $45 \mathrm{GPa}$ in shock pressure down to approximately $14 \mathrm{GPa}$ on release. A second tin sample at the same initial shock with a sapphire anvil undergoes a reshock to about $49 \mathrm{GPa}$ in pressure.

In addition to pyrometry results, we also obtained the reflectivity of lead and tin at $532 \mathrm{~nm}$ during these experiments. This not only provides us with valuable data on the "dynamic" target emissivity after shock excitation and release (or reshock) at the anvil interface, but also seems likely to reveal phase transitions. The reflectometry diagnostic that we used was derived from a recently developed method. ${ }^{8,9,10}$ This simplified version of the original diagnostic is only valid under specific conditions and assumptions (detailed below) but offers the advantage of being more compact and less costly than the earlier method with integrating spheres.

The experimental details (target assembly, diagnostics fielded, etc.) are described in section II. In section III, we describe the various diagnostics (VISAR, ${ }^{\mathrm{b}}$ pyrometry and reflectometry) and comment on the experimental configurations including the new reflectometry diagnostic. Finally, the results of the present investigation are summarized in section IV and discussed in section V.

\footnotetext{
${ }^{\mathrm{b}}$ Velocity interferometer system for any reflector.
} 


\section{EXPERIMENTAL DETAILS}

To minimize the number of experiments (and thus the cost), we used high explosive (HE) to drive large diameter flyer plates. ${ }^{11}$ The dimension of the Al6061 flyer plate $(300 \mathrm{~mm}$ in diameter) allowed us to place many samples in the same target baseplate. A schematic and pictures of the experimental assembly are provided in Figs. 2 through 6. A total of 10 metallic samples were mounted into the acrylic baseplate. These samples were located inside a 180-mm-diameter circle (see Fig. 4) where the curvature of the projectile plate is minimal. A common transmitter plate $(3.2 \mathrm{~mm}$ thick, $180 \mathrm{~mm}$ in diameter) transmits the shock from the flyer to the target assemblies. Additional smaller tungsten or tantalum transmitter plates (acting as shock attenuators) were placed between some of the samples and the common transmitter plate in order to achieve different shock pressures in the various targets. The release pressure of each sample was also dependent on the associated anvil window (LiF, PMMA or sapphire).

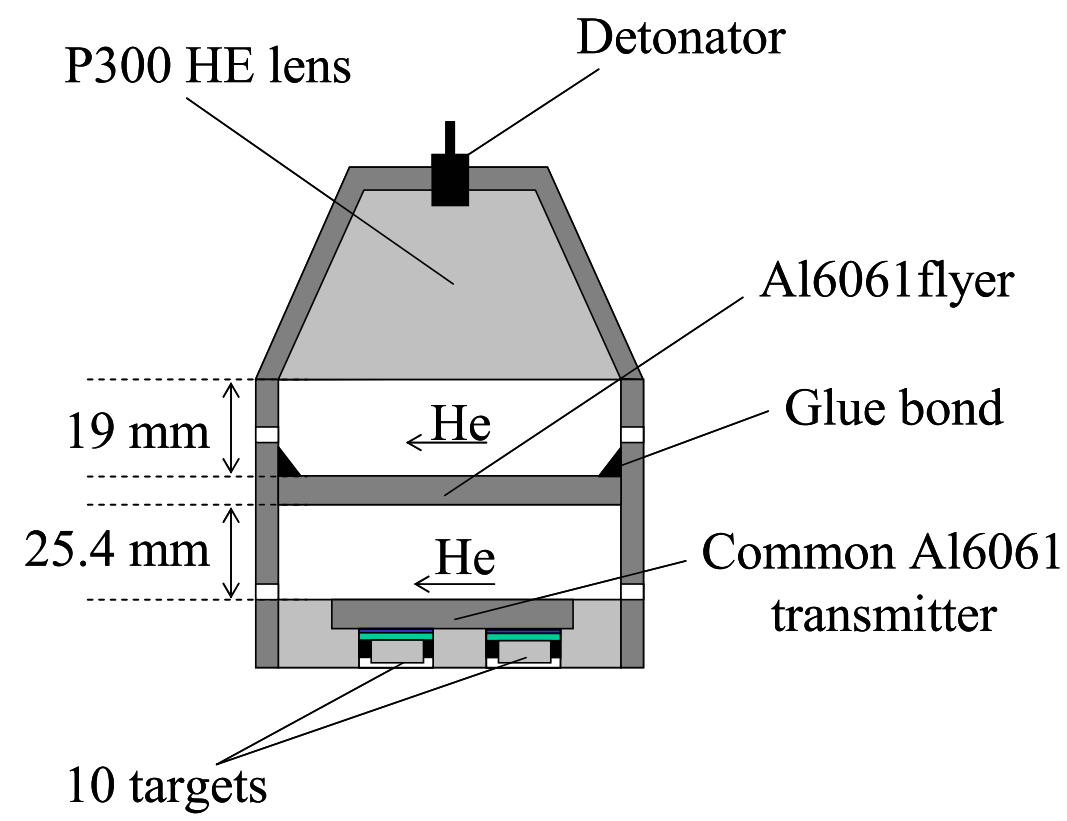


Fig. 2: Principle of the HE-driven flyer plate: the mass of P300 explosive was approximately $10 \mathrm{~kg}$. The flyer mass ranged from about 1.3 to $1.6 \mathrm{~kg}$, depending on the experiment. Helium is circulated inside the package to reduce the amplitude of the precursor shock that propagates through the gas.

The metallic samples were diamond-turned to obtain a mirror-like finish, and the transparent anvils were optically polished. The anvils were glued onto the samples to minimize interface effects (thermal diffusion, emission from shocked air, etc.). ${ }^{12}$ The glue (Loctite $^{\circledR}$ 326) has minimal absorption in the visible spectrum ${ }^{10,13}$ where the pyrometry and reflectometry measurements were performed. In Ref. 14, the authors used a composite anvil (made of two LiF windows glued together with Loctite ${ }^{\circledR} 358$, a glue made of similar chemical compounds as Loctite $\left.{ }^{\circledR} 326\right)$ to show that this glue remains transparent in the visible and near infrared spectrum up to (at least) $38 \mathrm{GPa}$ in shock pressure. We recently performed a similar experiment on Loctite ${ }^{\circledR} 326$ at $35 \mathrm{GPa}$ in shock pressure and did not detect any change in the glue transmittance (or refractive index) in the $600-800 \mathrm{~nm}$ wavelength range. We intend to perform additional experiments in the near future to study the behavior of this glue at higher pressures and over a larger wavelength range. For the present series of experiments, we will assume that the glue transmittance (in the ultraviolet and the visible spectrum) does not change up to at least $55 \mathrm{GPa}$ (which approximately corresponds to the highest pressure experienced by the glue in this study).

The target sample and anvil window diameters were 38 and $30 \mathrm{~mm}$, respectively. Their thicknesses (as well as those of the tantalum and tungsten transmitters if present) 
and that of the projectile flyer plate were individually selected to produce a steady

pressure at the sample-anvil interface for approximately $1 \mu$ s during the experiment. Each of the ten targets was associated with a single diagnostic: pyrometry, reflectometry or VISAR (see Fig. 3).
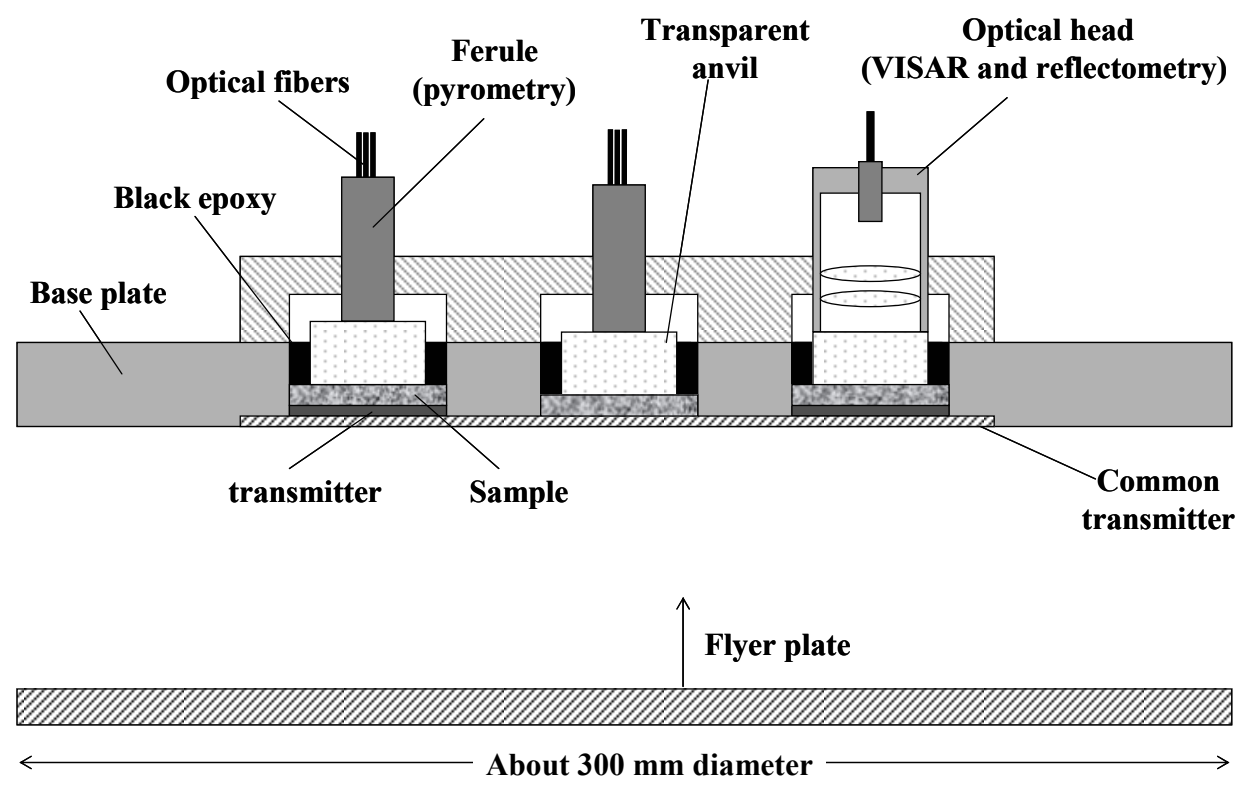

\section{Fig. 3: Experimental assembly (side view): the VISAR and reflectometry measurements were performed using a 2-lens optical head to control the laser signal return level. No optical head was used for the pyrometry measurements; the optical fibers were directly placed into contact with the transparent anvils.}

The $\mathrm{U}_{\mathrm{b}}$-block diagnostic ${ }^{15}$ (see Figs. 4 and 5) provided a measurement of the projectile plate velocity and curvature. The $U_{b}$-block diagnostic consists of a stepped aluminum transmitter plate facing the flyer plate and a Plexiglas window on the other side. A segmented gasket is placed between the shock transmitter and the Plexiglas window and argon gas is circulated through the gaps. When the projectile impacts the aluminum transmitter and the shock breaks out at the various heights in the stepped 
aluminum plate, the argon gas flashes and provides measurements of the shock arrival times at the various positions in the block. A rotating-mirror streak camera records the time history of the shock breakouts along the $\mathrm{U}_{\mathrm{b}}$-block length, and the images are processed to provide the projectile velocity. Three equally spaced streaks are imaged across the width of the $\mathrm{U}_{\mathrm{b}}$-block. If the projectile flyer plate has measurable curvature, the shock will appear first on one side of the $\mathrm{U}_{\mathrm{b}}$-block and will then sweep the three streaks successively, thus providing a measurement of the projectile curvature.

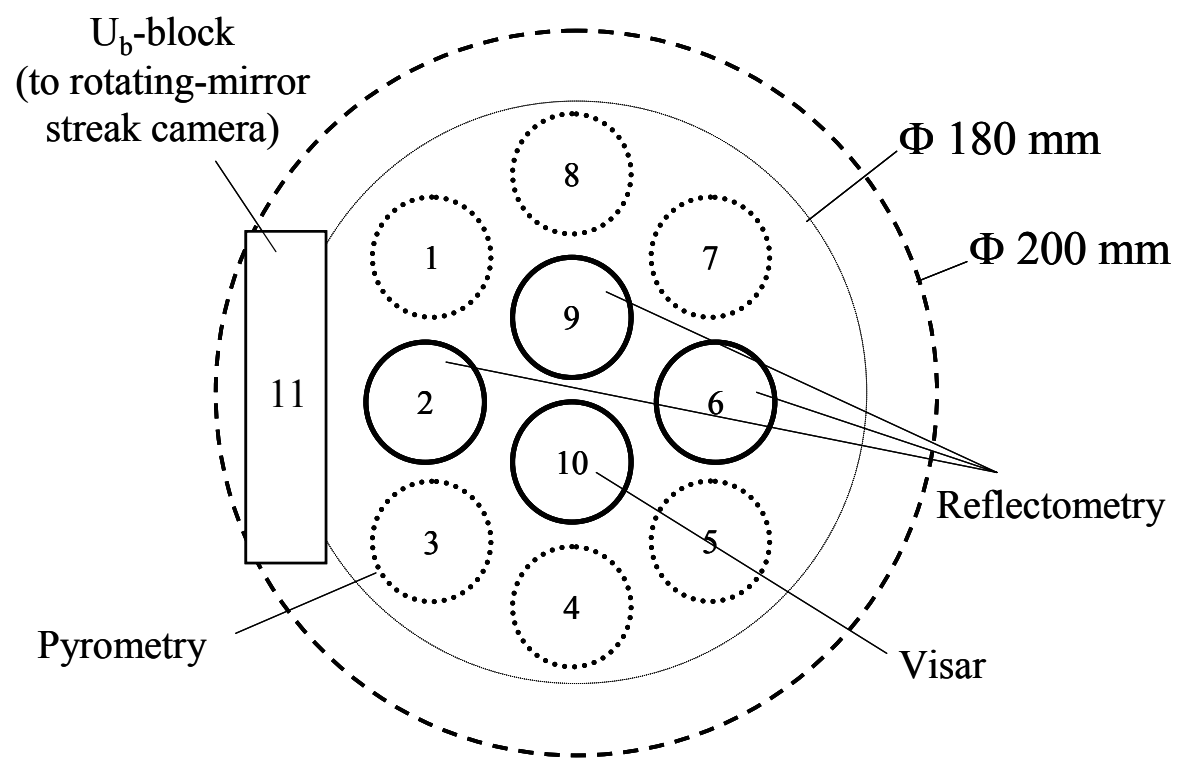

Fig. 4: Diagnostic arrangement on the target baseplate (top view): targets \#1, \#3, \#4, $\# 5$, \#7 and \#8 were assigned to pyrometry diagnostics. Reflectometry measurements were performed on targets \#2, \#6 and \#9. The velocity of the sample-anvil interface was measured on target \#10 using VISAR. 


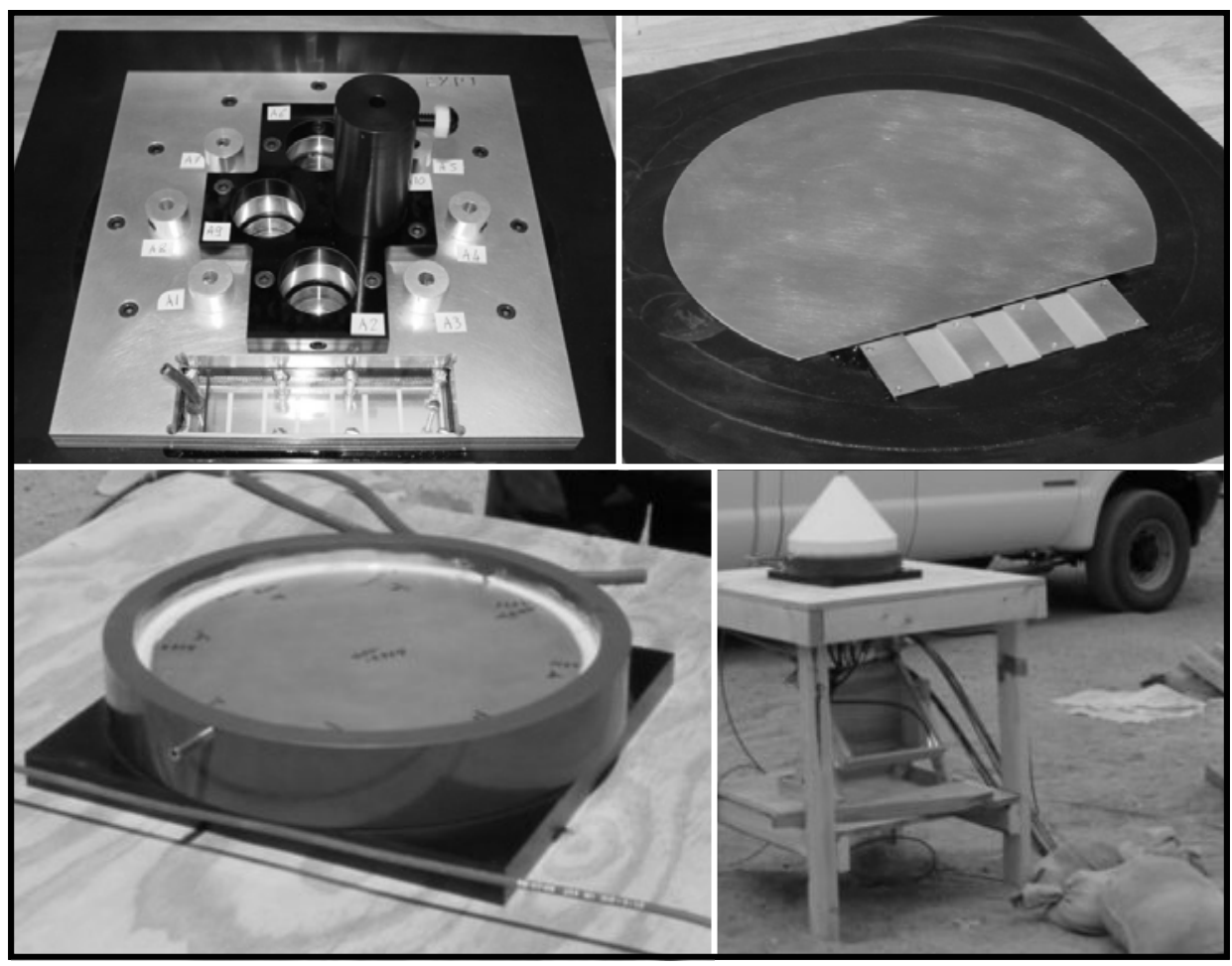

Fig. 5: Photographs of the experimental assembly. Top left photograph: the target baseplate (diagnostic side); top right photograph: the same baseplate (projectile side) showing the aluminum common transmitter and the $U_{b}$-block stepped transmitter; bottom left photograph: the flyer plate inside its barrel, placed onto the target baseplate (which is facing down); and bottom right photograph: the P300 HE lens placed onto the projectile barrel, and the first turning mirror (just below) used to relay the image of the $U_{b}$-block to the rotating-mirror streak camera. 


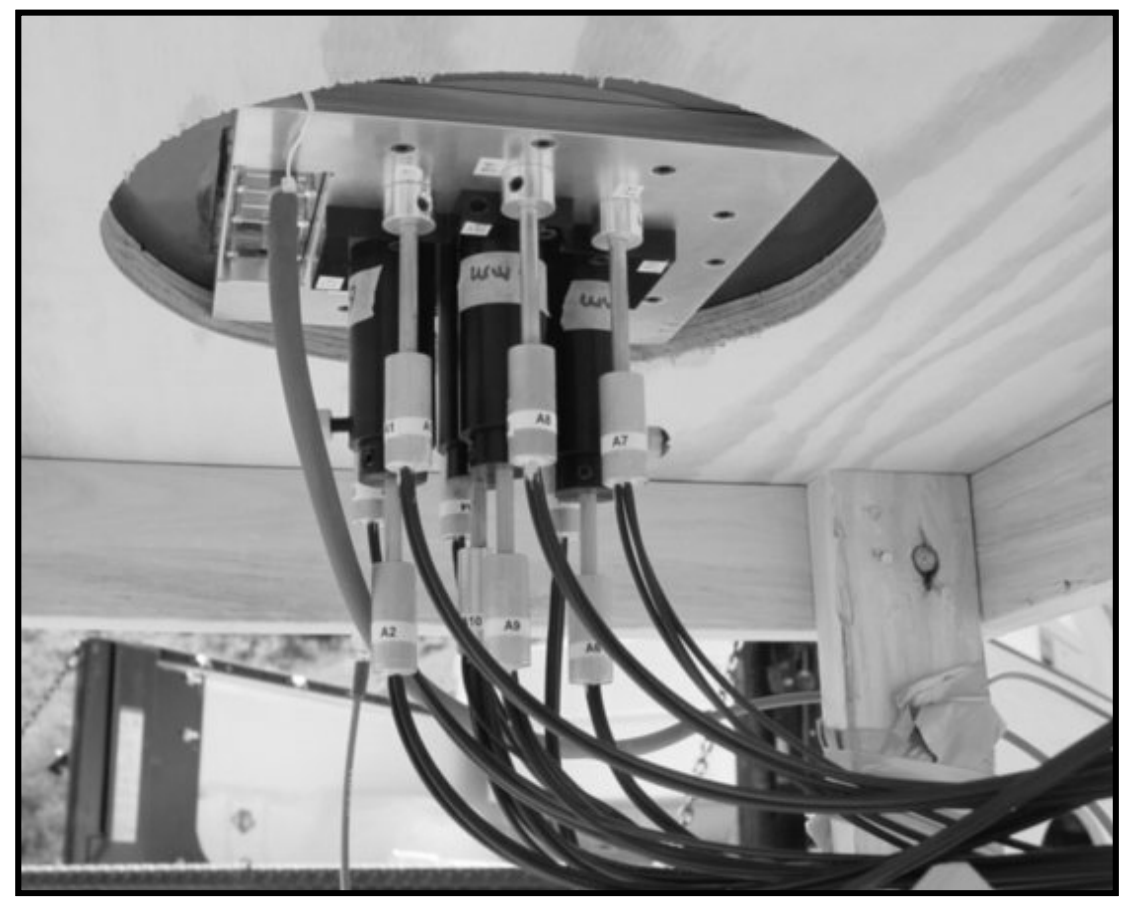

Fig. 6: Photograph of the diagnostic side of the target baseplate (facing down), in which all fiber probes are fielded and the argon gas is being circulated into the $U_{b^{-}}$ block.

\section{INTERFACE DIAGNOSTICS}

\section{III.1. VISAR diagnostic}

To perform the VISAR measurement of the velocity of the sample-anvil interface during the experiment, we used a probe made of $200-\mu$ m-diameter, silica fibers packed into a metallic needle ferule. The center fiber transmits the light and the peripheral fibers, packed into a single connector, collect the returning light signal. A 5-W continuous laser (operating at $532 \mathrm{~nm}$ in wavelength) illuminated the target for a $40-\mu$ s duration period shuttered with a Pockels cell. Because this laser was also used for the reflectometry measurements on three other targets on each package, the beam was split at the output of 
the Pockels cell and injected into four different fibers. The VISAR/reflectometry optical head was made of two $\mathrm{f} / 1 \mathrm{CaF}_{2}$ lenses $(25.4 \mathrm{~mm}$ in diameter) as illustrated in Fig. 7.

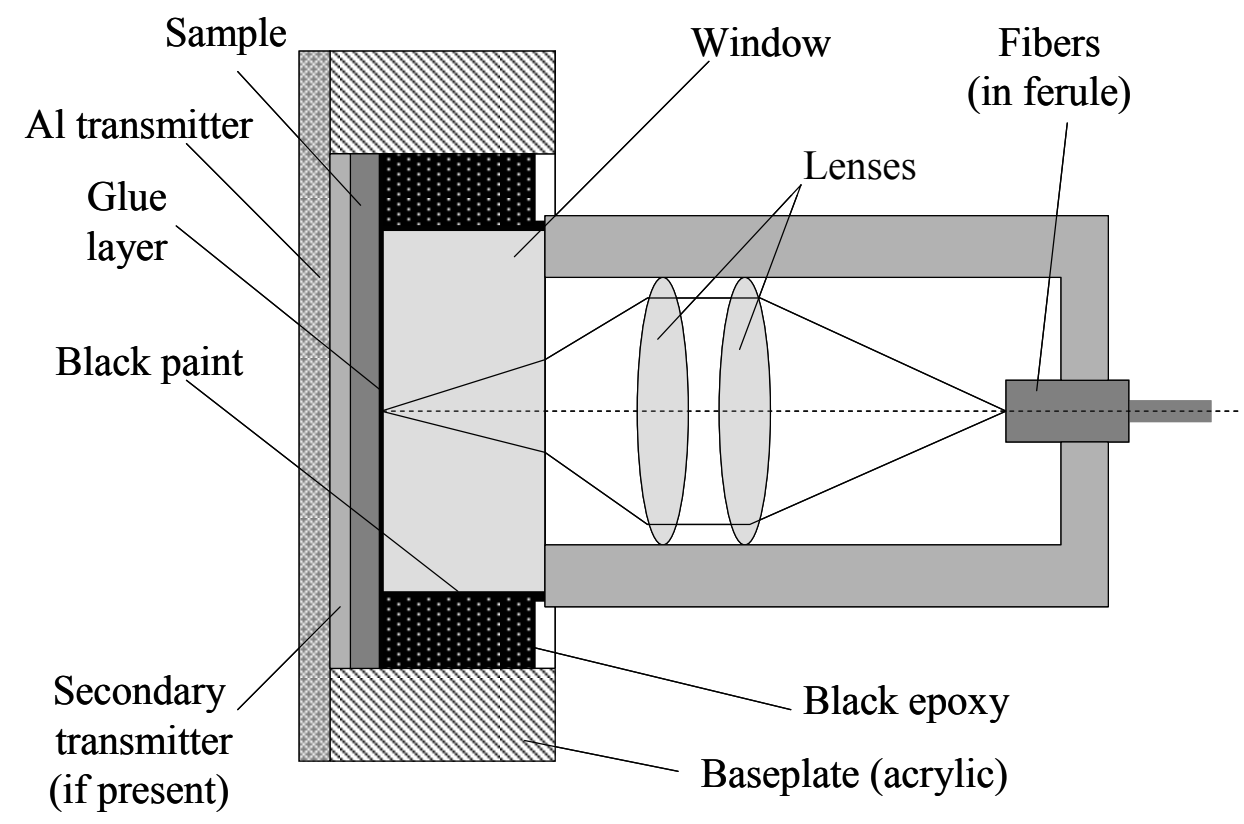

Fig. 7: Optical head used for the VISAR and reflectometry measurements.

Fig. 8 shows the VISAR data for the two shots from targets A10 and B10 (see section IV.1 for details on the targets). Note that the velocity profile shows an almost constant interface velocity (thus pressure) for approximately $1 \mu \mathrm{s}$ as expected, but the profile is not quite perfectly flat. There is a feature about $300 \mathrm{~ns}$ after shock emergence that may reflect some incipient damage in the flyer plate after acceleration. The plateau is not quite perfectly constant which may reflect the continuing HE gas acceleration of the flyer/target assembly after impact. 


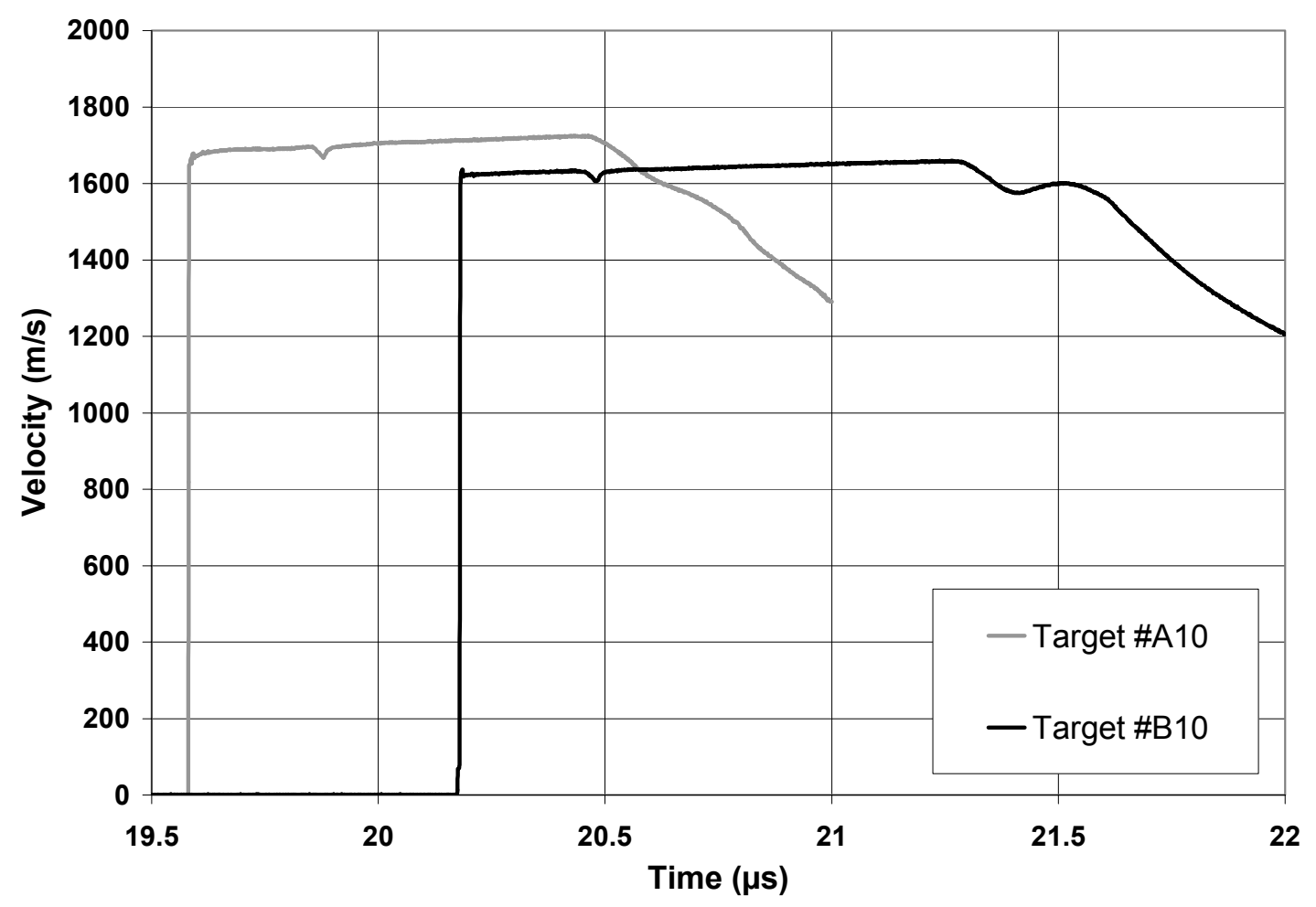

Fig. 8: VISAR data obtained from targets A10 and B10 (lead and tin, respectively, with LiF anvils).

\section{III.2. Pyrometry diagnostic}

In shock experiments, strong optical backgrounds originate outside the transparent anvil $^{9}$ and are usually caused by hot metal jets produced at the corners and singularities of the assembly. Optical background light is also produced when residual air near the measurement is shocked. To block these backgrounds, ${ }^{9}$ we coated the exterior of the transparent anvil with black acrylic paint and filled the gap around this window with black epoxy (see Figs. 3 and 7). The black epoxy consisted of carbon "lamp" black combined with a slow-curing epoxy. The epoxy was evacuated with a vacuum pump to minimize trapped air. 
The pyrometry measurements were performed using photomultiplier tubes (PMTs) each having a rise time of $\sim 5 \mathrm{~ns}$. Interference filters were used in each PMT to select wavelengths matched to the anticipated temperatures of the targets. We typically used filters operating in the ultraviolet and in the blue spectrum when the target temperature was expected to be higher than $2000^{\circ} \mathrm{C}$. Filters centered at longer wavelengths in the visible spectrum were selected for lower target temperatures. Each of the six pyrometry targets used two PMTs each with filters operating at distinct wavelengths, for a total of 12 pyrometry channels fielded in each experiment. Having more than one pyrometry channel per target was a prudent way to conduct the measurement (some fibers may be broken during fielding, some channels may saturate if temperatures were higher than expected, etc.). The pyrometry probes (see Fig. 9) were made of three 1-mm-diameter, high-OH silica fibers, with one fiber being a spare. Each bundle was $2.5 \mathrm{~m}$ long, coupled to $15 \mathrm{~m}$ fibers to relay the light to the PMT detectors.

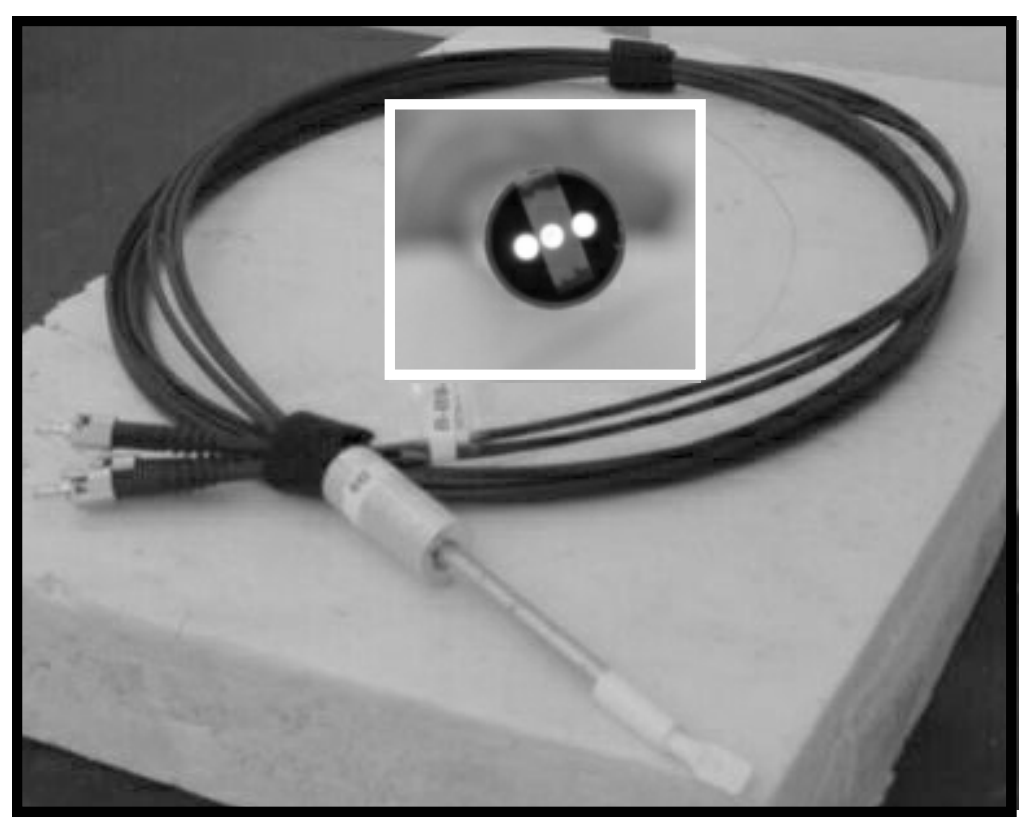

Fig. 9: Fiber bundles used for pyrometry and reflectometry measurements. Only two fibers were used for the pyrometry measurements, with the third as a spare. In 


\section{the case of reflectometry experiments, as illustrated in the center photograph, the center fiber, which was used to inject the laser beam, was covered with a thin optical diffuser to minimize the laser speckle.}

No optical head was associated to the pyrometry diagnostic: the fibers were placed in direct contact with the free surface of the transparent anvil, collecting light from approximately a 5-mm-diameter spot on the sample/window interface surface. The calibration, however, was performed using a calibration optical head made of one f/1 $\mathrm{CaF}_{2}$ lens (25.4 $\mathrm{mm}$ in diameter), in order to collect collimated light from the calibration light source. The optical transmittance of this additional lens was numerically accounted for in the effective pyrometer channel sensitivities. In pyrometry studies, the calibration source is usually a blackbody. However, electrical power, time and space were limiting factors in the bunker of our outdoor firing site. A blackbody calibration would have been difficult since we would have needed a very high-temperature blackbody. Instead, we used an integrating-sphere source, the radiance of which had been carefully calibrated with reference to a high-temperature blackbody. ${ }^{16}$

The pyrometry technique and the method for processing measured radiances into temperature are fully described in Ref. 9 (and references therein). Suitable choice for the pyrometry wavelengths (with respect to the expected temperatures) minimizes the effect on temperature from the large uncertainties in the dynamic emissivities of the target. For example, if one assumes an allowed range of 0.1 to 1 , which is reasonable for the emissivity of many metals in the visible spectrum, it is possible to obtain the sample 
temperature with 10 to $20 \%$ uncertainty (see numerical example provided in Fig. 5 of Ref. 9).

In the present experiments, where only two pyrometry channels were associated with each target, the issue is that the temperature range that can be measured is rather limited. The PMTs can operate properly from $\sim 50 \mathrm{mV}$ up to only a few volts (their linearity limit). In the case of lead, this voltage range corresponds to temperature coverage of only $200-300 \mathrm{~K}$ for the lowest target temperatures ( $\sim 1500 \mathrm{~K}$ with the PMMA anvils) and up to a maximum of $800-900 \mathrm{~K}$ for the highest target temperatures ( $\sim 3000 \mathrm{~K}$ with the sapphire anvils). In other words, not only do the theoretical predictions of temperature need to be as accurate as possible, but our initial guesses for the dynamic emissivities must also be accurate enough since the actual emissivities directly shift the range of true temperatures covered by the pyrometry channels. Similarly, if the actual projectile flyer velocities are significantly different from what is expected, this can also have unfavorable consequences on the measurements.

Pyrometry measurements through LiF and PMMA do not present any particular difficulties. LiF is commonly used in pyrometry studies (and remains transparent up to at least $160 \mathrm{GPa}$ in shock pressure ${ }^{17}$ ) and although PMMA has not yet been used extensively, it can be used successfully at pressures below its transparency threshold. ${ }^{18}$ However, sapphire emits light when shocked above its elastic limit (see Refs. 13 and 19, and references therein). Therefore, instead of observing constant radiance at the sampleanvil interface after shock emergence (typically for one microsecond), the radiance increases approximately linearly with time (Fig. 10). Such data were processed according to the method discussed in Ref. 13 which allows inferring the thermal contribution from 
the metallic sample and isolating the light emitted by the sapphire anvil. Note, however, that even though the radiances increase by 35 to $40 \%$ between $20.7 \mu$ s and $22 \mu$ s in Fig. 10, the corresponding "apparent" temperature (radiance temperature) only increases from approximately $3000 \mathrm{~K}$ to $3100 \mathrm{~K}$, i.e., less than $5 \%$ in relative value.

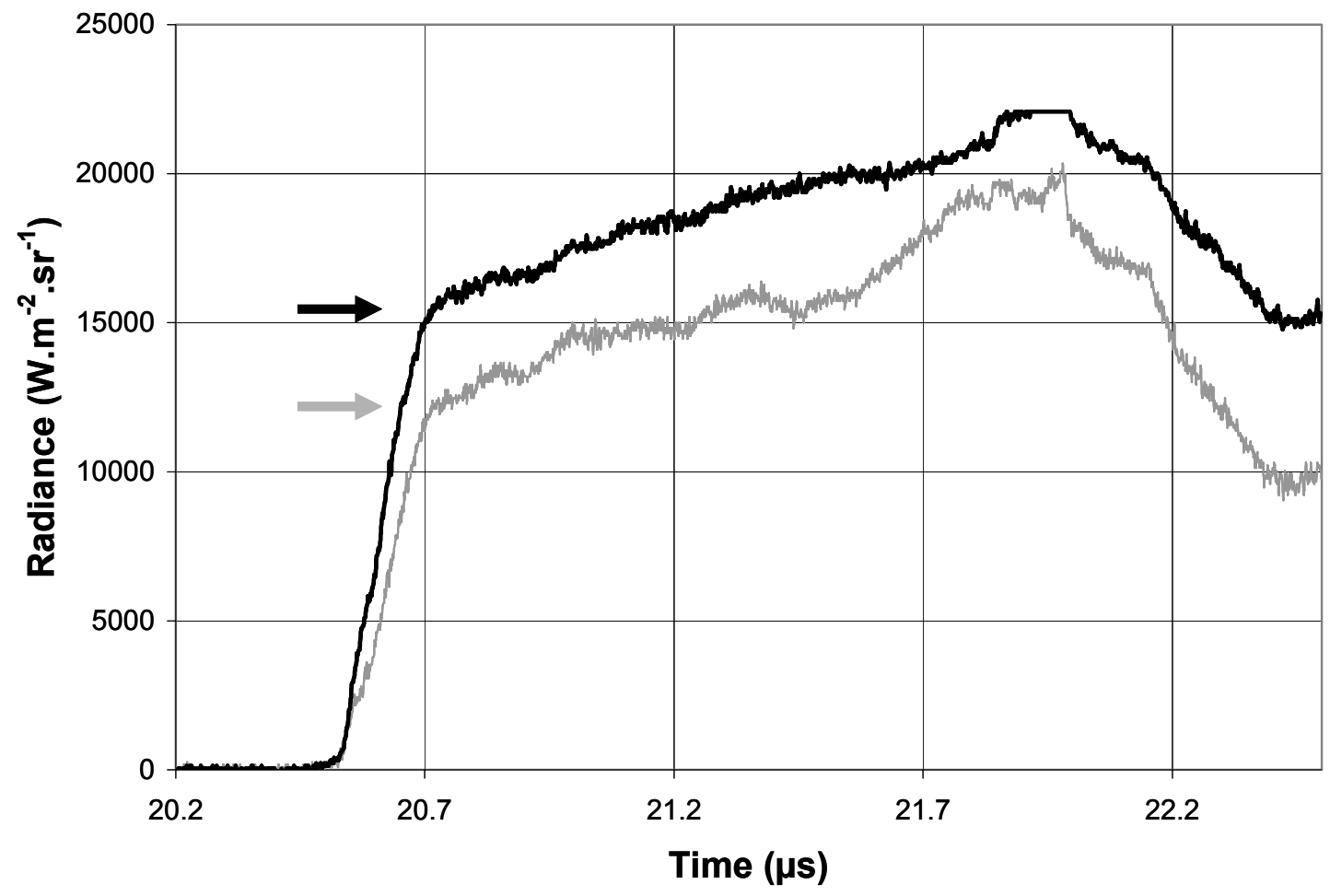


Fig. 10: Radiances obtained from one of the lead-sapphire targets, at $510 \mathrm{~nm}$ (black curve) and $460 \mathrm{~nm}$ (grey curve) in wavelengths. The filter bandwidths, at $50 \%$ of the peak transmittance, were 87 and $74 \mathrm{~nm}$, respectively. The rising shape of the signal from $20.7 \mu$ s until approximately $22 \mu$ s is due to light emission from sapphire, as the shock travels through it and compresses a progressively larger volume of material. The thermal contribution from the metallic sample approximately corresponds to levels indicated by the horizontal arrows and is inferred from a graphical method which is described in full detail in Ref. 13.

\section{III.3. Reflectometry diagnostic}

When studying metals, pyrometry accuracy is mainly limited by the fact that the emissivity of the shock-loaded sample is not known precisely. We recently developed a technique based on integrated reflectometry to measure the sample emissivity during the experiment (dynamic emissivity). ${ }^{8,9,10}$ Initially we fielded a simplified version of this diagnostic to obtain qualitative data and possibly detect phase transitions.

\section{III.3.1. Conceptual description of the diagnostic}

For the sake of discussion, suppose that the diagnostic is fielded as illustrated in Fig. 11. No coupling lens is used and the fibers are directly in contact with the LiF window. The center fiber is connected to a laser and illuminates the metallic sample which has a mirror-like finish. To minimize the laser-induced speckle, a diffuser ${ }^{\mathrm{c}}{ }^{\text {is }}$

\footnotetext{
${ }^{\mathrm{c}}$ We used a thin plastic diffuser, Roscolux ${ }^{\circledR}$ model \#116 - tough white diffusion. These filters are designed for use in theatrical lighting projectors and can sustain moderately high temperatures.
} 
placed on the transmit fiber as illustrated in Fig. 9. The reflected spot covers the fiber bundle with an approximately uniform radiance (thanks to the diffuser) and light can be collected by the peripheral receive fibers which are connected to PMTs. Since the light spot covering the fiber bundle is reasonably uniform, this approach makes the diagnostic insensitive to any reasonable tilt in the projectile (and thus, the bundle is less susceptible to the effects of the shock).

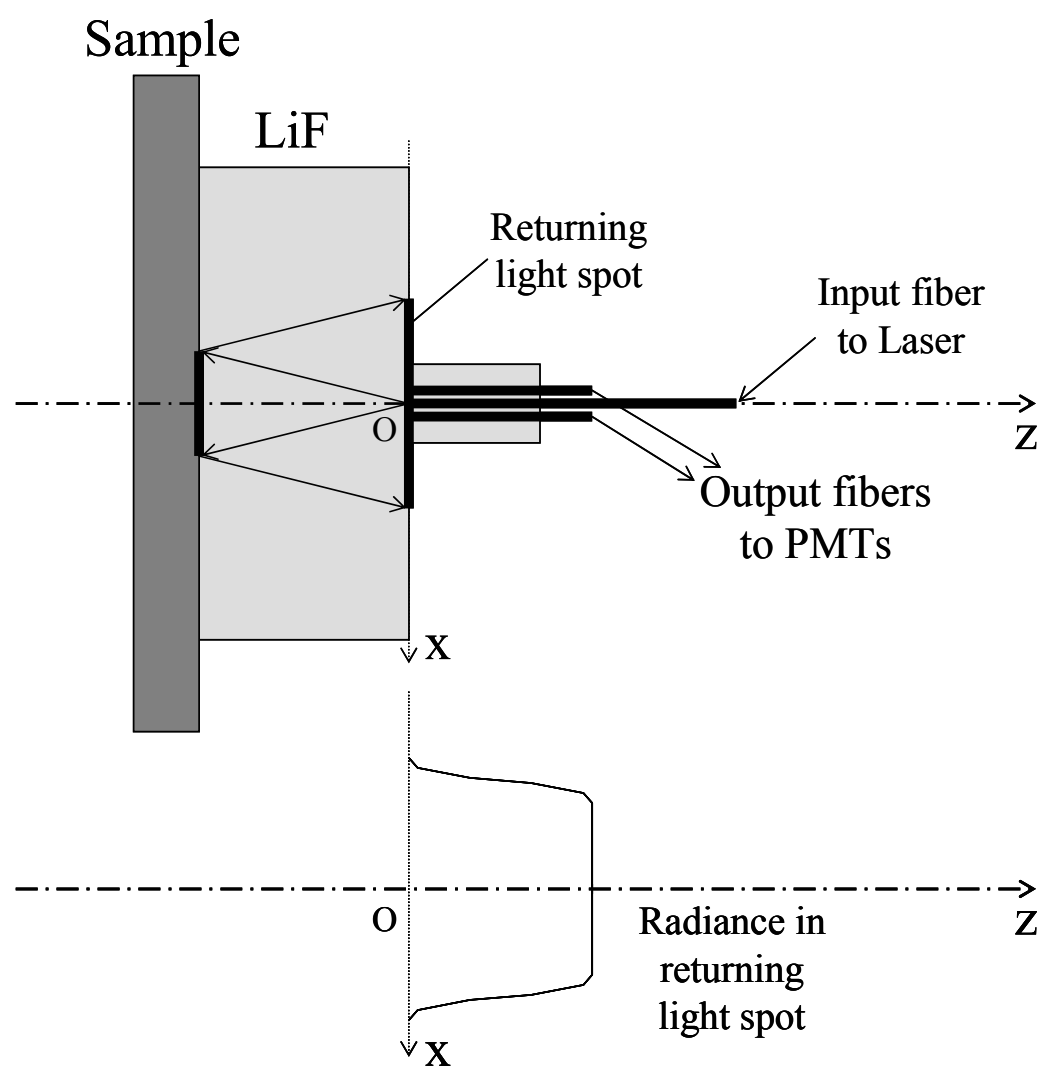

Fig. 11: Principle of the reflectivity diagnostic. A thin plastic diffuser is placed in contact with input fiber to minimize laser speckle and obtain a uniform return spot.

When the shock breaks out at the metal-LiF interface, a change in the signal level can come from several effects: a) a change in the surface roughness, b) a change in the index of refraction and internal transmission of the glue or the LiF window, or c) a 
change in the metal intrinsic emissivity (due to compression and/or phase transitions). According to previous results on bismuth, ${ }^{18}$ we believe that during the experiment the sample roughness remains imprinted in the glue (which does not melt in the investigated pressure range, assuming the Hugoniot of Loctite ${ }^{\circledR} 326$ is the same as that of the common Epon ${ }^{\circledR} 828$ epoxy $^{20}$ ). In other words, the surface of the metallic sample is assumed to remain mirror-like (or at least smooth in case of lead) during the experiment, even when the metal melts. Since the surface finish is expected to remain stable, we will assume in this study that the roughness of the sample does not contribute to the change in the reflectivity signal during the experiments.

When shock-compressed, the transparent anvil ( $\mathrm{LiF}$ ) remains transparent up to the megabar pressure range ${ }^{17}$ but a change in refractive index occurs. This variation, however, remains negligible at these pressures. ${ }^{21}$

As mentioned in section II, between 600 and $800 \mathrm{~nm}$ in wavelength, the transmittance (and refractive index) of Loctite ${ }^{\circledR} 326$ does not appear to change up to (at least) $35 \mathrm{GPa}$ in shock pressure (which exceeds the pressure levels experienced by the glue in all targets associated with reflectometry measurements). For the present study (and until more data are available), we will assume that this property is also valid down to the shortest wavelength used in the present work (i.e., $400 \mathrm{~nm}$ ).

For these reasons, the only variation that is assumed to be detected by the diagnostic is the relative change in intrinsic emissivity/reflectivity of the metallic sample when the shock breaks out at the interface.

This datum, along with a preliminary measurement of the initial absolute static reflectivity of the target can allow one to infer the absolute reflectivity of the target 
during the experiment. Finally, one can calculate the reflectivity of the metal-LiF interface (and directly infer the emissivity of this interface) from the absolute (total) reflectivity of the target (metallic sample + glue + window), by accounting for the multiple refections of light rays into the $\mathrm{LiF}$ anvil (see Ref. 8).

Again, this is only valid when a transparent anvil is glued onto a mirror-like metallic sample. In more general configuration, implying no anvil or a rougher metal surface state, it is necessary to field an integrating sphere and use the exact method. ${ }^{8}$

\section{III.3.2. Actual configuration of the diagnostic}

The optical configuration illustrated in Fig. 11 is a simple conceptual example that allows us to describe the principle of the method, though it is not optimal for coupling laser light back to the PMTs, especially because of the diffuser which produces a large diameter returning spot. Instead, we used the same optical head as that described in paragraph III.1. for VISAR (see Fig. 7), but moved away from the target by a distance $d$ (see Fig. 12, where $d$ was set to $6 \mathrm{~mm}$ ) so that the returning spot diameter was $\sim 9-$ $10 \mathrm{~mm}$ on the collecting fibers, increasing the level of laser light collected for reflectivity measurements. 


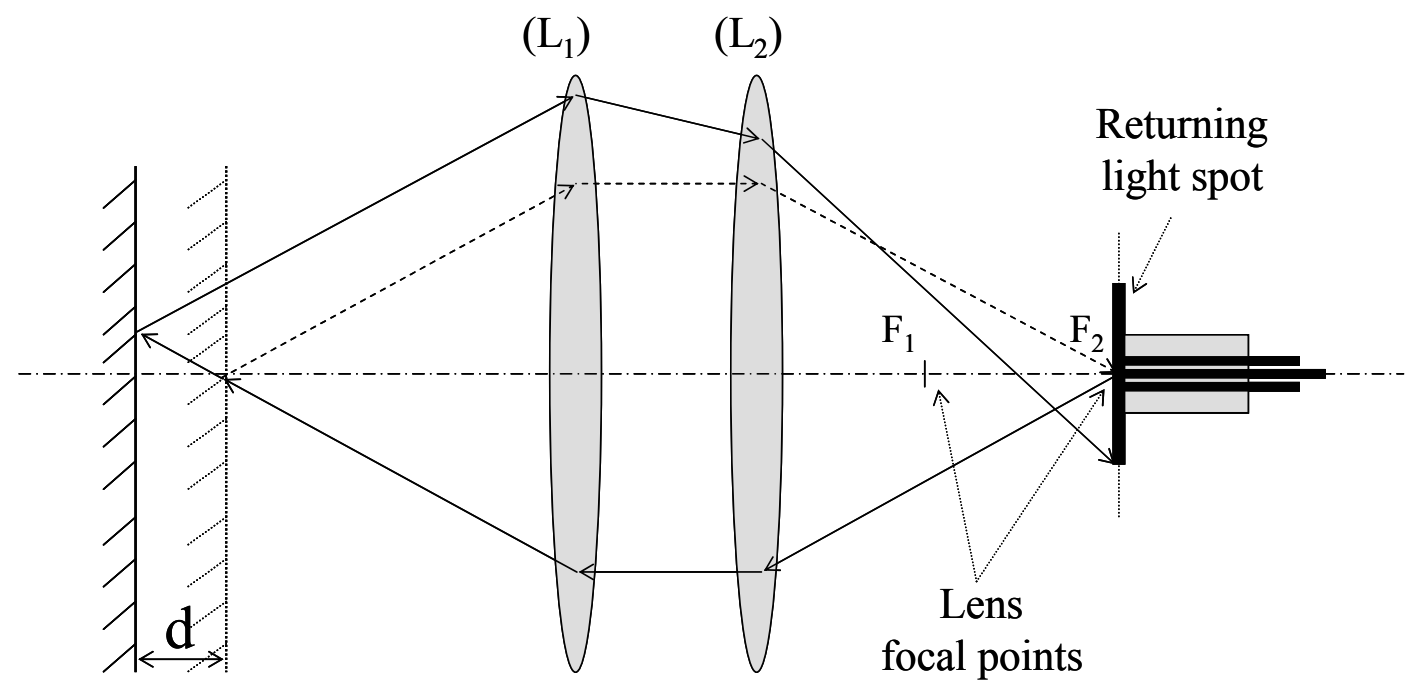

Fig. 12: Actual optical configuration used for reflectivity measurements.

The photomultipliers used for reflectivity measurements were similar to those used for pyrometry except that their electronic bases were modified so that they could drive more current during the $40-\mu$ s-long laser pulse. The fiber probes were the same as the ones used for pyrometry (1-mm-diameter fibers), except that a diffuser was glued onto the injecting fiber as illustrated in Fig. 8. Optical filters at $532 \mathrm{~nm}$ (either 3 or $10 \mathrm{~nm}$ wide) were used with these photomultipliers to limit the wavelength range of the detected light.

Using narrow 532-nm-wavelength filters eliminated, or at least reduced, the thermal contribution from the hot target in the reflectometry signal, thus making the change in target reflectivity the major contribution to this measurement. Based on the preliminary calibrations of the reflectometry channels (using a bright uniform radiance source), this setup was insensitive to thermal light originating from the metallic sample up to approximately $2000^{\circ} \mathrm{C}$ in surface temperature. The contribution is $\sim 10 \mathrm{mV}$ at 
$2000^{\circ} \mathrm{C}$. At $2500^{\circ} \mathrm{C}$ and $3000^{\circ} \mathrm{C}$ the thermal contribution would be $\sim 100 \mathrm{mV}$ and $\sim 1000 \mathrm{mV}$, respectively.

When the target temperature is higher than about $2000^{\circ} \mathrm{C}$, it is best to use a twochannel differential diagnostic as illustrated in Fig. 13 (which is what we actually fielded). The two channels differ in the width of the 532-nm-wavelength filters used; one filter was $10 \mathrm{~nm}$ wide and the second was $3 \mathrm{~nm}$ in bandwidth. Assuming that the temperature and emissivity of the target is uniform over the sample surface, the two measured voltages (after the shock breaks through the sample-LiF interface) can be written as follows: $\mathrm{V}_{1}=\mathrm{S}_{\mathrm{T}}+\mathrm{S}_{\mathrm{R}}$ and $\mathrm{V}_{2}=a \mathrm{~S}_{\mathrm{T}}+b \mathrm{~S}_{\mathrm{R}}$, where $\mathrm{S}_{\mathrm{T}}$ is the thermal contribution from the target and $S_{R}$ is the level of reflected laser light that we want to measure. A preliminary calibration of this two-channel diagnostic is needed to accurately measure the coefficients $a$ and $b$ (as represented in Fig. 13, $a$ is the ratio of both channel sensitivities to a given thermal signal; similarly, $b$ is the ratio of both channel sensitivities to a given laser input signal). This calibration is performed by placing a bright uniform radiance source in front of the two fibers with no laser light. The ratio of the voltages obtained in the two channels gives the coefficient $a$. The coefficient $b$ is obtained from the shot data, just before the shock breaks through the sample-anvil interface since no thermal light is emitted by the sample before this time. Note that if both channels had the same sensitivity at $532 \mathrm{~nm}$ (same photomultiplier sensitivity, same filter transmittance, same fiber transmittance), the coefficient $b$ would be equal to 1 , and the coefficient $a$ would be equal to 0.3 (which is the ratio of the two 532-nm filter bandwidths). However, since we associated the more sensitive PMTs to the 3-nm-wide filters (to balance the channels), the coefficient $a$ was closer to 1 in the present series of experiments. Using this calibration, 
the experimental voltages obtained from both channels can be processed to produce both the thermal contribution $\mathrm{S}_{\mathrm{T}}$ and reflectivity signal $\mathrm{S}_{\mathrm{R}}$.

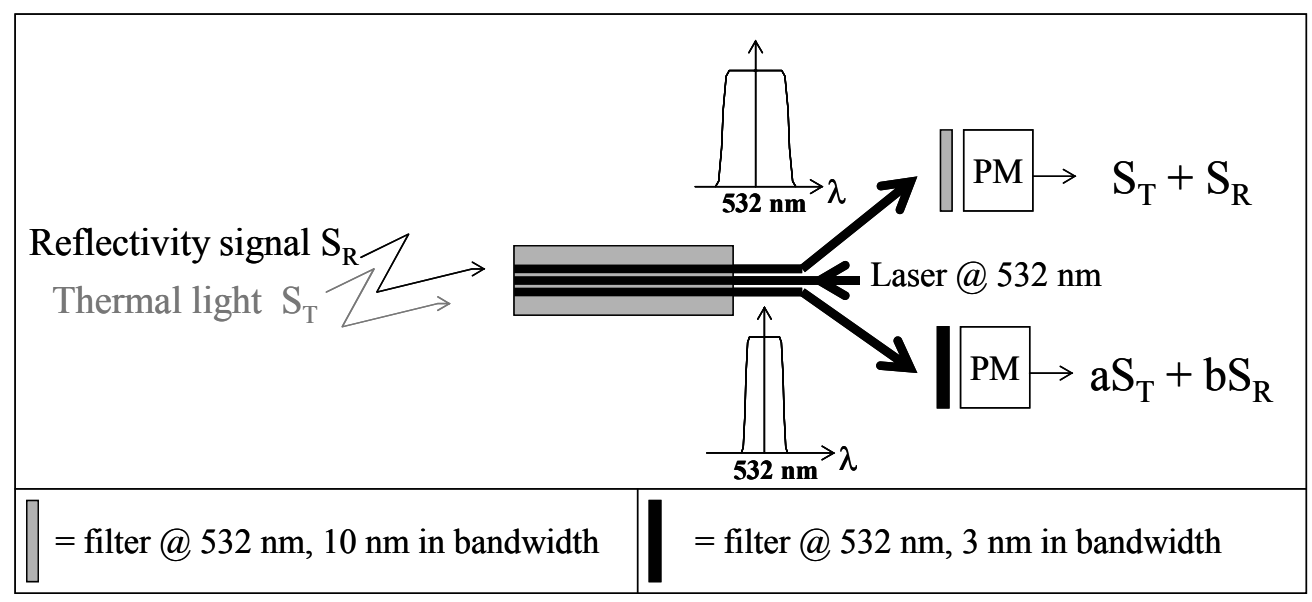

Fig. 13: Principle of the two-channel diagnostic.

\section{III.3.3. Validation of the diagnostic}

Before carrying out the experiments on lead, we needed to validate the concept of the two-channel diagnostic on a simple gas-gun experiment. We selected tin as proof-test material for several reasons: a) one can produce a nicely uniform mirror finish on this material, b) the low-pressure solid-solid phase transition (white-tin to body-centered tetragonal lattice- " $\beta$ to $\mathrm{BCT}$ "3) would produce a small but perhaps detectable change in reflectivity, and c) since this phase transition takes place at low pressure (and temperature), very little thermal light would contribute to the signals detected by both reflectometry channels. The result of this experiment is shown in Fig. 14, where the advantage of having more than one reflectivity channel is evident. Two fibers, each at different locations on the reflected light spot, provide the same result, confirming that there were no serious problems during the experiment (such as a large tilt in the shock, or variation in the reflectivity across the surface). 


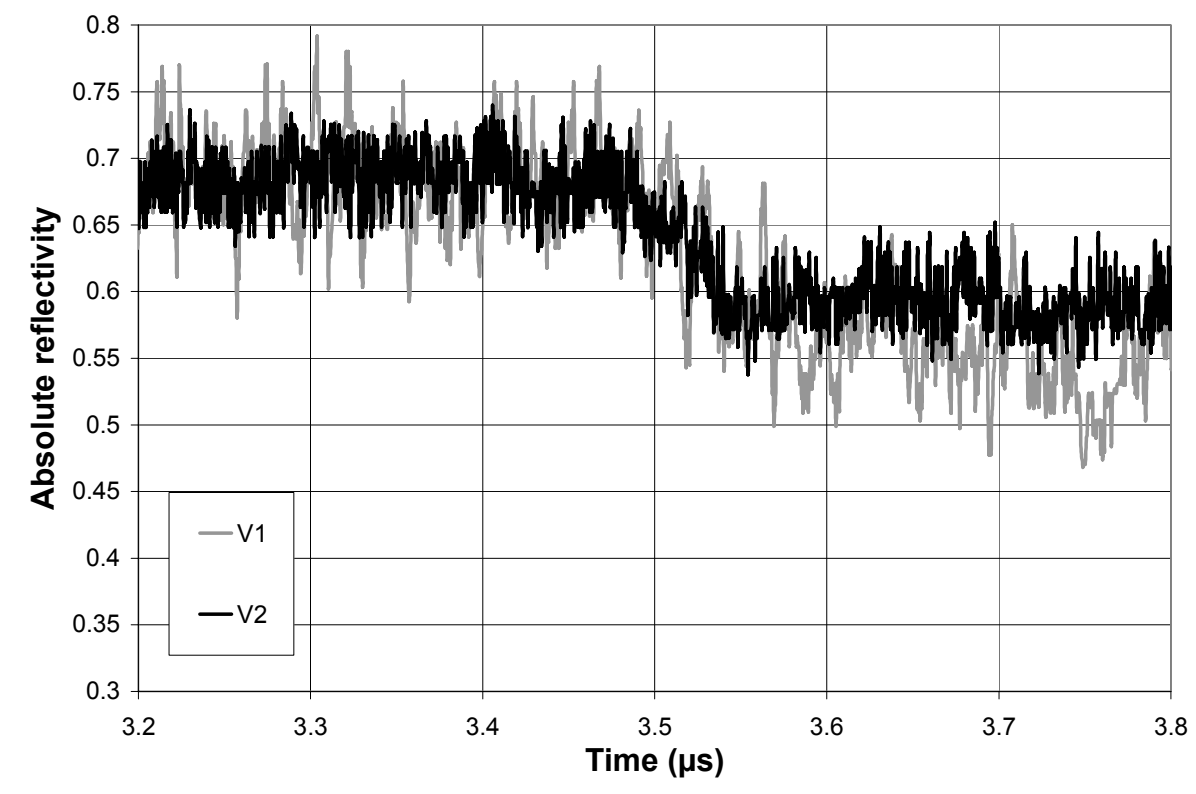

Fig. 14: Result of the validation experiment. The shock pressures in tin and LiF were 9 and 7 GPa, respectively.

\section{EXPERIMENTAL RESULTS}

\section{IV.1. Description of the experiments and packages}

We performed two experiments (referred as experiments \#A and \#B respectively). The HE/flyer packages were designed so that the flyer would impact the common transmitter at approximately $3.7 \mathrm{~km} / \mathrm{s}$ during the first experiment and at $3.4 \mathrm{~km} / \mathrm{s}$ during the second, the flyer plates being $6.35 \mathrm{~mm}$ and $7.37 \mathrm{~mm}$ thick, respectively. The target characteristics are summarized in Tables 1 and 2: transmitter, sample, and anvil materials, target initial reflectivity (total reflectivity of the sample and anvil glued together, at $655 \mathrm{~nm}$ in wavelength and at approximately $20^{\circ}$ of incidence angle), and the diagnostic fielded. A rough estimate of the initial reflectivity of the target was obtained using a laser pointer as a light source and a photometer as a sensor. The reflectivity was 
measured at the center of the target. The photometer detector was $10 \mathrm{~mm}$ in diameter and coupled to a f/1, 25-mm-diameter lens. Such an optical configuration can collect light from the target over a fairly large solid angle. This is important since a set of parallel light beams are produced in the transparent anvil window from multiple reflections. By using such a lens, most of the flux produced in this pattern was collected. Obviously, these data can only be considered as semi-quantitative since we likely lost part of the diffuse light (especially in case of lead, for which the results are surely underestimated). An integrating-sphere technique ${ }^{22}$ would give much more accurate values. Furthermore, the initial reflectivity measurement was at $655 \mathrm{~nm}$ (instead of $532 \mathrm{~nm}$ where the shock measurements were performed) because no green laser was available in our labspace at the time (and green laser pointers appear to be less stable in power). From Tables 1 and 2, the average static reflectivity of lead was 0.34 . The average reflectivity for the tin targets was 0.74 .

The surface finish $\left(\mathrm{R}_{\mathrm{a}}\right)$ of the tin samples was about $0.011 \mu \mathrm{m}$ and that of the lead sample was about $0.028 \mu \mathrm{m}$. The tin purity was 0.9998 . A very small amount of calcium ( $0.07 \%$ by weight) was added when casting the lead (initially 0.995 in purity). The addition of calcium hardens the metal and more easily permits machining a mirror finish without inducing a significant change to the lead equation of state. The $\mathrm{LiF}$ was ultraviolet grade, monocrystalline and $<100>$ in crystal orientation. The sapphire was monocrystalline and c-cut ( $\{0001\}$ crystal orientation $)$. The PMMA was regular amorphous polymethylmethacrylate of high quality (aquarium grade).

The transmitter and sample thicknesses ranged from 2.5 to $3.5 \mathrm{~mm}$ depending on the target. All the transparent anvil windows were $15 \mathrm{~mm}$ in thickness. 
Table 1: First assembly data

\begin{tabular}{|c|c|c|c|c|c|}
\hline Target \# & Transmitter & Sample & Anvil & Target reflectivity & Diagnostic \\
\hline A1 & none & lead & LiF & $32 \%$ & Pyrometry \\
\hline A2 & none & lead & LiF & $35 \%$ & Reflectometry \\
\hline A3 & none & lead & PMMA & $33 \%$ & Pyrometry \\
\hline A4 & none & lead & sapphire & $34 \%$ & Pyrometry \\
\hline A5 & tantalum & lead & LiF & $28 \%$ & Pyrometry \\
\hline A6 & tantalum & lead & LiF & $31 \%$ & Reflectometry \\
\hline A7 & tantalum & lead & PMMA & $32 \%$ & Pyrometry \\
\hline A8 & tantalum & lead & sapphire & $32 \%$ & Pyrometry \\
\hline A9 & none & tin & LiF & $74 \%$ & Reflectometry \\
\hline A10 & none & lead & LiF & $37 \%$ & VISAR \\
\hline
\end{tabular}

Table 2: Second assembly data

\begin{tabular}{|c|c|c|c|c|c|}
\hline Target \# & Transmitter & Sample & Anvil & Target reflectivity & Diagnostic \\
\hline B1 & none & lead & LiF & $38 \%$ & Pyrometry \\
\hline B2 & none & lead & LiF & $34 \%$ & Reflectometry \\
\hline B3 & none & lead & PMMA & $32 \%$ & Pyrometry \\
\hline B4 & none & lead & sapphire & $36 \%$ & Pyrometry \\
\hline B5 & none & lead & LiF & $33 \%$ & Pyrometry \\
\hline B6 & none & tin & LiF & $74 \%$ & Reflectometry \\
\hline B7 & none & tin & PMMA & $76 \%$ & Pyrometry \\
\hline
\end{tabular}




\begin{tabular}{|c|c|c|c|c|c|}
\hline Target \# & Transmitter & Sample & Anvil & Target reflectivity & Diagnostic \\
\hline B8 & none & tin & sapphire & $73 \%$ & Pyrometry \\
\hline B9 & tungsten & lead & LiF & $42 \%$ & Reflectometry \\
\hline B10 & none & tin & LiF & $75 \%$ & VISAR \\
\hline
\end{tabular}

\section{IV.2. General comments on the results and the data processing}

As mentioned earlier, only one laser source was used to perform the VISAR and reflectivity measurements, leading to little available light power. As a result, some of the reflectometry data are rather noisy (see Fig. 15 for instance). Also, the reflectivity of lead was not uniform over its surface, due to a first-stage oxidation. Just after being diamondturned, the lead samples were stored inside a vacuum container until we glued the anvils onto them (approximately a week later). Because lead tends to oxidize quickly, some faint white spots, randomly scattered on the sample surface, were apparent to the eye before the gluing, although too faint to be visible in a photograph. We left a few spare samples in contact with air for two months until the oxidation spots would turn black (and until we glued LiF windows to "freeze" the surface state, see Fig. 16): the long term oxidation is clearly nonuniform and the glue appears to chemically react with lead and/or its oxide(s). Measurements of the integrated reflectivity of these oxidized/glued samples were performed over a large wavelength range (UV-VIS-IR) ${ }^{23}$ and compared to the reflectivity of bare lead samples from the same lot. These measurements showed that no unexpected and dramatic loss in reflectivity takes place in the visible spectrum, but we will, however, process the pyrometry data obtained for lead in a cautious way (see below). 
The first consequence of this nonuniformity is that two reflectivity channels can (and did) provide different results even though they were fielded on the same target. In Fig. 15 two channels viewed two different parts of the lead target B9 (with probably two different initial reflectivity values) and thus provided two somewhat different values for the relative variation of reflectivity.

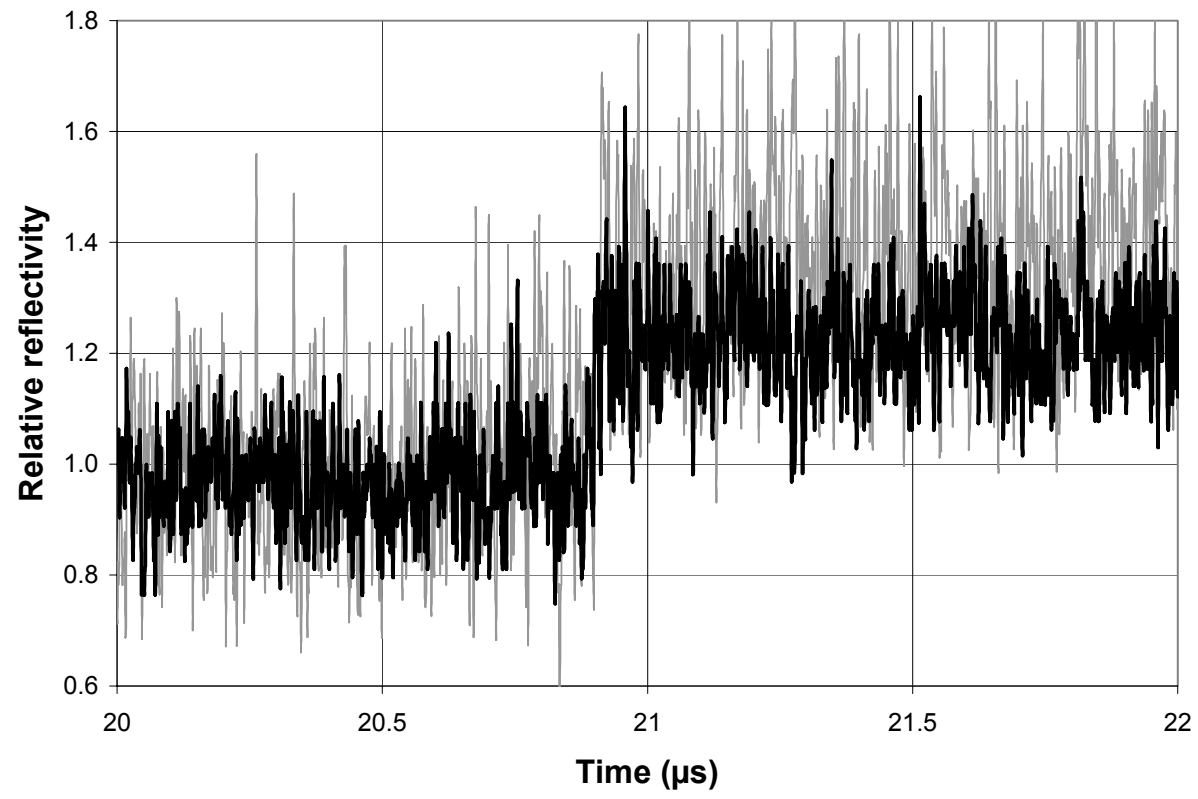

Fig. 15: Results from target $B 9$ (lead and LiF). Both channels appear to show somewhat different changes in reflectivity.

The second consequence is that, for very hot targets with thermal light contributing substantially to the signal, there is no way to calculate and isolate this contribution. The approach developed in section III.3.2. is indeed not applicable since the two reflectivity channels are viewing two different reflectivity contributions $S_{R 1}$ and $S_{R 2}$, as well as and two different thermal contributions $\mathrm{S}_{\mathrm{T} 1}$ and $\mathrm{S}_{\mathrm{T} 2}$ (the emissivity also being non-uniform). 
Fortunately, most of our targets were not hot enough to generate significant thermal contributions in the reflectivity channels. Target A6 (lead-LiF), however, produced a thermal contribution of $\sim 10-15 \mathrm{mV}$ on both reflectivity channels, according to their calibration (with a bright integrating sphere source) and the target experimental temperature (obtained from target A5, which experienced the same pressure history). This thermal contribution was neglected in the data processing, and consequently the results from this target must be regarded cautiously (i.e., the reflectivity for A6 is somewhat underestimated).

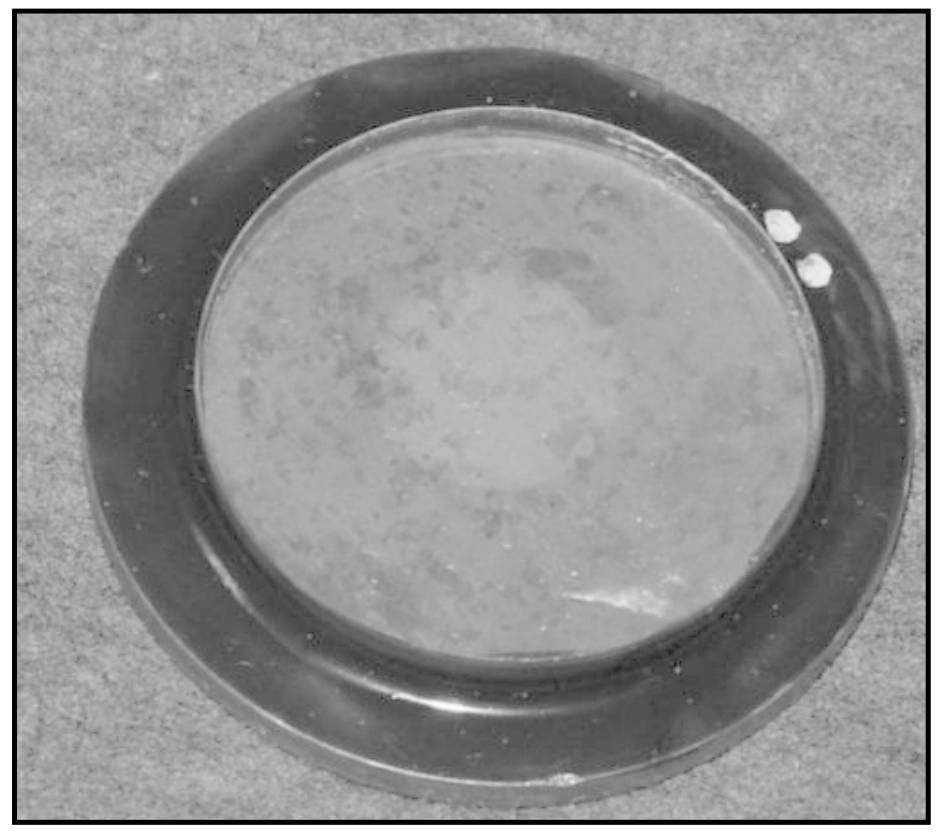

Fig. 16: A lead sample was intentionally left in contact with air for two months to promote surface oxidation. A thin $\mathrm{LiF}$ window was glued onto the sample afterward. Note that the oxidation is nonuniform and that the glue (Loctite ${ }^{\circledR}$ 326) also appears to chemically react slightly with lead and/or its oxide(s). The white spot in the middle appears to have been caused by the drop of glue developing from the instant it was placed onto the lead sample with the $\mathrm{LiF}$ window covering it until the time it 


\section{was centered in the mechanical press and pressed. This feature was not apparent on the shot targets, probably because the lead did not have time to oxidize to as significant an extent.}

On average we note that the reflectivity of the lead targets tended to increase (up to $40 \%$ ) during these dynamic experiments (see Fig. 15 for instance), while the reflectivity of the tin targets tended to decrease (typically by $25 \%$, see Fig. 17 for example). No previous data on lead is available, but some for shock-loaded tin (in contact with $\mathrm{LiF}$ ) are available ${ }^{9}$ and also indicate that reflectivity of tin decreases under comparable levels of compression.

According to these dynamic results and the initial reflectivities of the targets (Tables 1 and 2), we processed the pyrometry radiances into temperatures using the assumptions that the emissivity of the tin targets remained between 0.1 and 0.6 at measured wavelengths during the experiments (see Ref. 9 and references therein for a detailed description of the pyrometry technique). In the case of experiments with lead, we assumed that emissivity remained between 0.2 and 1 for these wavelengths, which is very conservative with respect to the measured dynamic reflectivities. Because of the nonuniformity in lead reflectivity (mentioned above), we indeed believe that it was a prudent way to proceed, at least until more information is available (in particular, a theoretical study of the reflectivity of shock-loaded lead is in progress). 


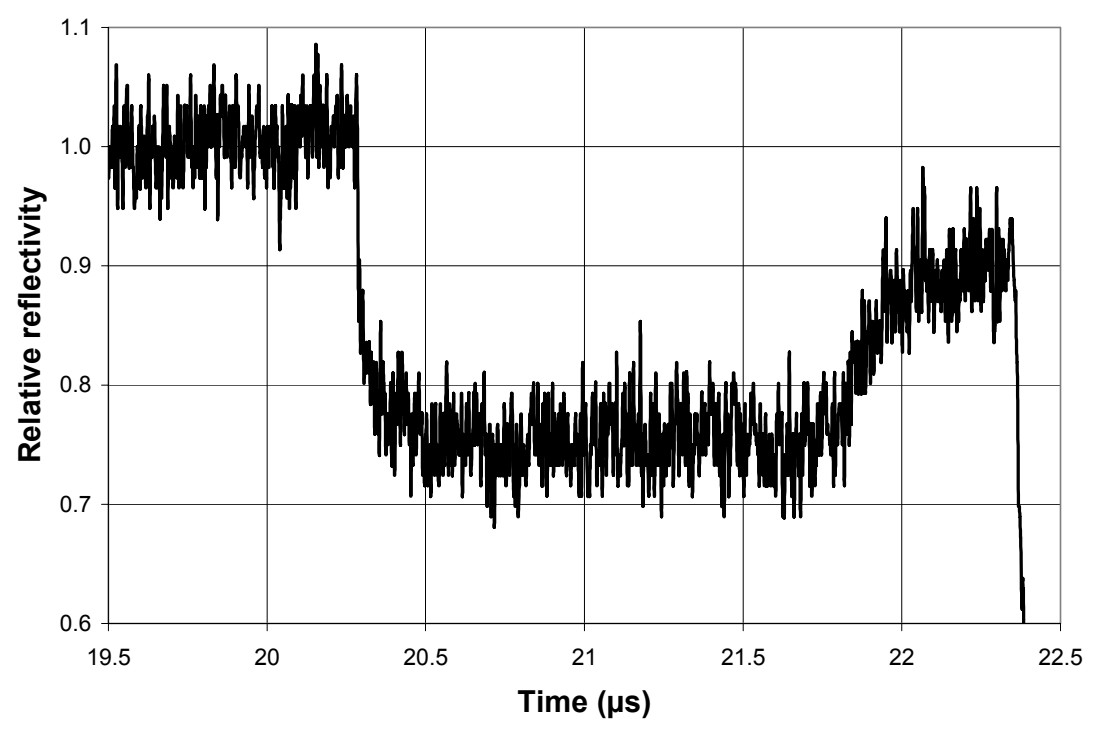

Fig. 17: Results from target $\mathrm{B6}$ (tin and LiF). Tin exhibits a decrease in reflectivity when the shock breaks through at the tin-LiF interface (at about $20.3 \mu \mathrm{s}$ ) and takes the material into the solid-liquid coexistence region (according to our estimates). When the fan of release waves (coming from the rear of the projectile) decreases the pressure at the tin-LiF interface (between approximately $21.7 \mu$ s and $22.1 \mu$ s), tin turns fully liquid (according to our estimates) and its reflectivity increases. Finally, at about $22.4 \mu \mathrm{s}$, the shock breaks out at the free surface of $\mathrm{LiF}$, ending the measurement.

\section{IV.3. Experimental results}

The experimental results are shown in Tables 3 and 4: the shock pressure in the sample, the interface pressure (i.e., shock pressure in the metal/anvil interface), diagnostics results for the relative changes in target reflectivity ( $R 1$ and $R 2$ ), and the target interface temperature $(\mathrm{T})$ or the target interface velocity $(\mathrm{V})$. Note that R1 and R2 are relative changes in the reflectivity at $532 \mathrm{~nm}$ recorded on both channels of the 
reflectometry diagnostic. All data were processed within the first $300 \mathrm{~ns}$ after shock emergence at the sample-window interface.

For the first experiment, the projectile velocity (from the $\mathrm{U}_{\mathrm{b}}$-block diagnostic) could not be measured with a reasonable uncertainty due to improper setting of the optical aperture in the rotating-mirror camera (the streaks were brighter than expected and the traces on the film were too thick). The VISAR data provided $1.69 \mathrm{~km} / \mathrm{s}$ in velocity at the lead-LiF interface (target A10, see Table 3), which corresponds to $3.73 \mathrm{~km} / \mathrm{s}$ in flyer velocity (i.e., consistent with the expected velocity: $3.7 \mathrm{~km} / \mathrm{s}$ ).

For the second experiment, the projectile velocity measured from the $\mathrm{U}_{\mathrm{b}}$-block diagnostic was $3.39( \pm 0.02) \mathrm{km} / \mathrm{s}$. The measured flyer bow indicated that the pyrometry targets (which are located on the largest diameter) did not experience more than 45 milliradian angle of projectile tilt during this experiment. The VISAR data provided $1.63 \mathrm{~km} / \mathrm{s}$ in velocity at the tin-LiF interface (target B10, see Table 4) which corresponds to $3.38 \mathrm{~km} / \mathrm{s}$ in flyer velocity (and is consistent with both the expected velocity, $3.4 \mathrm{~km} / \mathrm{s}$, and the measured one, $3.39 \mathrm{~km} / \mathrm{s}$ ).

As indicated in the tables, some of these results were not satisfactory: pyrometry data from target B8 (tin-sapphire) were discarded because we did not properly set up the channel (insufficient optical density) and the signal exceeded the linear dynamic range of the PMTs. One of the reflectivity channels from target B6 (tin-LiF) was discarded because of its unusual shape, probably due to a bright spot emitting in the field of view of this fiber (likely to have been caused by a defect on the sample surface or an air bubble trapped in the glue). One of the reflectivity channels from target B9 (tin-LiF) was not used because the laser input signal was too low to provide a reasonable signal-to-noise 
ratio. Finally, as mentioned in paragraph IV.2., the reflectometry results obtained from target A6 (lead-LiF) may be underestimated because the target radiance may have thermally contributed to both reflectometry signals.

The calculation of the shock and interface pressures was inferred from the VISAR results (and associated uncertainties). Because of the symmetrical initial A16061-A16061 impact, mass velocity in the shocked common transmitter is half of the flyer velocity. For A16061, lead, tin, tungsten, tantalum, LiF, PMMA and sapphire, experimental shock Hugoniot data ${ }^{20,24}$ were used and the release isentropes of lead, tin, tungsten and tantalum were assumed to be the mirror images of corresponding shock-loci. This well-known approximation $^{25}$ seems appropriate for these materials in the investigated pressure range because these phase transitions do not exhibit too large a volume change. For the secondshock Hugoniot of Al6061, we made the same assumption and checked its validity (successfully) using a theoretical aluminum equation of state. Because the experimental Hugoniot data of PMMA and (especially) sapphire exhibit significant scatter, we accounted for this dispersion in the calculation of the interface pressures (Tables 3 and 4; the corresponding additional uncertainties are 0.15 and $0.55 \mathrm{GPa}$, respectively).

\section{Table 3: Experimental results (first assembly)}

\begin{tabular}{|c|c|c|c|c|}
\hline Exp. \# & Sample & $\begin{array}{c}\text { Shock pressure } \\
\text { in the sample }\end{array}$ & $\begin{array}{c}\text { Interface } \\
\text { pressure }\end{array}$ & Diagnostic \\
& & results \\
\hline A1 & lead & $57.1( \pm 0.6) \mathrm{GPa}$ & $33.1( \pm 0.3) \mathrm{GPa}$ & $\mathrm{T}=2180( \pm 140) \mathrm{K}$ \\
\hline A2 & lead & $57.1( \pm 0.6) \mathrm{GPa}$ & $33.1( \pm 0.3) \mathrm{GPa}$ & $\mathrm{R}_{1}=114( \pm 4) \%$ \\
& & & $\mathrm{R}_{2}=112( \pm 5) \%$ \\
\hline
\end{tabular}




\begin{tabular}{|c|c|c|c|c|}
\hline Exp. \# & Sample & $\begin{array}{c}\text { Shock pressure } \\
\text { in the sample }\end{array}$ & $\begin{array}{c}\text { Interface } \\
\text { pressure }\end{array}$ & $\begin{array}{c}\text { Diagnostic } \\
\text { results }\end{array}$ \\
\hline A3 & lead & $57.1( \pm 0.6) \mathrm{GPa}$ & $14.4( \pm 0.3) \mathrm{GPa}$ & $\mathrm{T}=1670( \pm 100) \mathrm{K}$ \\
\hline A4 & lead & $57.1( \pm 0.6) \mathrm{GPa}$ & $53.5( \pm 1) \mathrm{GPa}$ & $\mathrm{T}=3390( \pm 370) \mathrm{K}$ \\
\hline A5 & lead & $48.6( \pm 0.5) \mathrm{GPa}$ & $28.7( \pm 0.3) \mathrm{GPa}$ & $\mathrm{T}=2150( \pm 140) \mathrm{K}$ \\
\hline A6 & lead & $48.6( \pm 0.5) \mathrm{GPa}$ & $28.7( \pm 0.3) \mathrm{GPa}$ & $\mathrm{R}_{1}=110( \pm 4) \%$ \\
& & & & $\mathrm{R}_{2}=127( \pm 3) \%$ \\
\hline A7 & lead & $48.6( \pm 0.5) \mathrm{GPa}$ & $12.3( \pm 0.3) \mathrm{GPa}$ & $\mathrm{T}=1490( \pm 110) \mathrm{K}$ \\
\hline A8 & lead & $48.6( \pm 0.5) \mathrm{GPa}$ & $46.7( \pm 1) \mathrm{GPa}$ & $\mathrm{T}=3100( \pm 280) \mathrm{K}$ \\
\hline A9 & tin & $51.2( \pm 0.5) \mathrm{GPa}$ & $35.9( \pm 0.3) \mathrm{GPa}$ & $\mathrm{R}_{1}=75( \pm 7) \%$ \\
& & & & $\mathrm{R}_{2}=$ discarded \\
\hline A10 & lead & $57.1( \pm 0.6) \mathrm{GPa}$ & $33.1( \pm 0.3) \mathrm{GPa}$ & $\mathrm{V}=1.69( \pm 0.01) \mathrm{km} / \mathrm{s}$ \\
\hline
\end{tabular}

Table 4: Experimental results (second assembly)

\begin{tabular}{|c|c|c|c|c|}
\hline Exp. \# & Sample & $\begin{array}{c}\text { Shock pressure } \\
\text { in the sample }\end{array}$ & $\begin{array}{c}\text { Interface } \\
\text { pressure }\end{array}$ & $\begin{array}{c}\text { Diagnostic } \\
\text { results }\end{array}$ \\
\hline B1 & lead & $49.7( \pm 0.5) \mathrm{GPa}$ & $29.3( \pm 0.3) \mathrm{GPa}$ & $\mathrm{T}=2170( \pm 140) \mathrm{K}$ \\
\hline B2 & lead & $49.7( \pm 0.5) \mathrm{GPa}$ & $29.3( \pm 0.3) \mathrm{GPa}$ & $\begin{array}{c}\mathrm{R}_{1}=109( \pm 4) \% \\
\mathrm{R}_{2}=100( \pm 3) \%\end{array}$ \\
\hline B3 & lead & $49.7( \pm 0.5) \mathrm{GPa}$ & $12.6( \pm 0.3) \mathrm{GPa}$ & $\mathrm{T}=1560( \pm 50) \mathrm{K}$ \\
\hline B4 & lead & $49.7( \pm 0.5) \mathrm{GPa}$ & $47.6( \pm 1) \mathrm{GPa}$ & $\mathrm{T}=3210( \pm 290) \mathrm{K}$ \\
\hline B5 & lead & $49.7( \pm 0.5) \mathrm{GPa}$ & $29.3( \pm 0.3) \mathrm{GPa}$ & $\mathrm{T}=2120( \pm 100) \mathrm{K}$ \\
\hline
\end{tabular}




\begin{tabular}{|c|c|c|c|c|}
\hline Exp. \# & Sample & $\begin{array}{c}\text { Shock pressure } \\
\text { in the sample }\end{array}$ & $\begin{array}{c}\text { Interface } \\
\text { pressure }\end{array}$ & $\begin{array}{c}\text { Diagnostic } \\
\text { results }\end{array}$ \\
\hline B6 & tin & $44.7( \pm 0.4) \mathrm{GPa}$ & $31.6( \pm 0.3) \mathrm{GPa}$ & $\begin{array}{c}\mathrm{R}_{1}=76( \pm 3) \% \\
\mathrm{R}_{2}=\text { Discarded }\end{array}$ \\
\hline B7 & tin & $44.7( \pm 0.4) \mathrm{GPa}$ & $14.4( \pm 0.3) \mathrm{GPa}$ & $\mathrm{T}=1400( \pm 50) \mathrm{K}$ \\
\hline B8 & tin & $44.7( \pm 0.4) \mathrm{GPa}$ & $49.3( \pm 1) \mathrm{GPa}$ & Discarded \\
\hline B9 & lead & $37.4( \pm 0.4) \mathrm{GPa}$ & $22.6( \pm 0.2) \mathrm{GPa}$ & $\mathrm{R}_{1}=124( \pm 5) \%$ \\
& & & & $\mathrm{R}_{2}=138( \pm 9) \%$ \\
\hline B10 & tin & $44.7( \pm 0.4) \mathrm{GPa}$ & $31.6( \pm 0.3) \mathrm{GPa}$ & $\mathrm{V}=1.63( \pm 0.01) \mathrm{km} / \mathrm{s}$ \\
\hline
\end{tabular}

\section{DISCUSSION}

Fig. 18 summarizes the pyrometry results obtained for lead in the present investigation. The extrapolation of the low-pressure static melting curve (cf. Refs. 2 and 3) from $9 \mathrm{GPa}$ to $12.3 \mathrm{GPa}$ (the lowest PMMA interface pressure) gives $1400-1450 \mathrm{~K}$ to be compared with the value $1490( \pm 110) \mathrm{K}$ obtained in our shock experiment. Consequently, this point is probably on the melting curve but the PMMA point corresponding to the highest interface pressure is perhaps slightly above (its initial shock pressure being somewhat higher than expected). Since our dynamic points are on the melt curve or in its immediate vicinity (according to the arguments given in part I), it follows that at high pressure the true melting curve of lead is somewhat above the one given by laser-heated diamond-anvil cell experiments ${ }^{1}$ and is not very different from the theoretical curve proposed in Ref. 6. 
A refined calculation of this melt curve (with a new calculation of the Hugoniot) and the corresponding detailed analysis of present experimental results are in progress and will be reported soon. ${ }^{26}$ This improved analysis takes into account both the metal cooling at the interface with the anvil (even if the glue acts as a thermal insulator) and the increase of interface temperature due to the series of shock-reshock occurring inside the glue layer. ${ }^{27}$ This last phenomenon is particularly important in the case of a lead-sapphire target because the shock-impedances of these materials are much higher than that of the glue. However, as our experimental points are in the solid-liquid coexistence region, the consequence of both effects is only a variation of the solid-liquid proportion.

It is interesting to note in Fig. 18 that the point obtained at about $33 \mathrm{GPa}$ (with a $\mathrm{LiF}$ anvil) appears somewhat inconsistent with the two LiF points nearby. However, since the measured signals do not indicate a problem in this target during the experiment, this inconsistency is unexplained at this time.

The simplified version of the reflectometry diagnostic proved very useful, giving strong indications on the probable values of sample emissivities during the experiment. Consequently, it is easier for us to make more reliable assumptions on the range of allowed emissivity values and thus to calculate the temperature from the measured radiances with higher degree of confidence. However, because of its tendency to oxidize, leading to a nonuniform surface reflectivity, lead does not appear to be an ideal candidate for reflectivity measurements if the target gets hot enough $\left(\mathrm{T}>2000^{\circ} \mathrm{C}\right.$ in this experiment) to thermally contribute to the signal. In such cases, the approach developed in section III.3.2. is no longer valid and the thermal contribution cannot be numerically isolated from the reflectivity contribution. 
When adequate return signal light is available (see Fig. 17 for instance), this diagnostic may be rather sensitive to pressure-induced phase transitions, which are likely to induce the leading contribution in the observed changes of reflectivity. However, the amplitude of this contribution necessarily depends on the considered metal and the wavelength at which the measurement is performed.

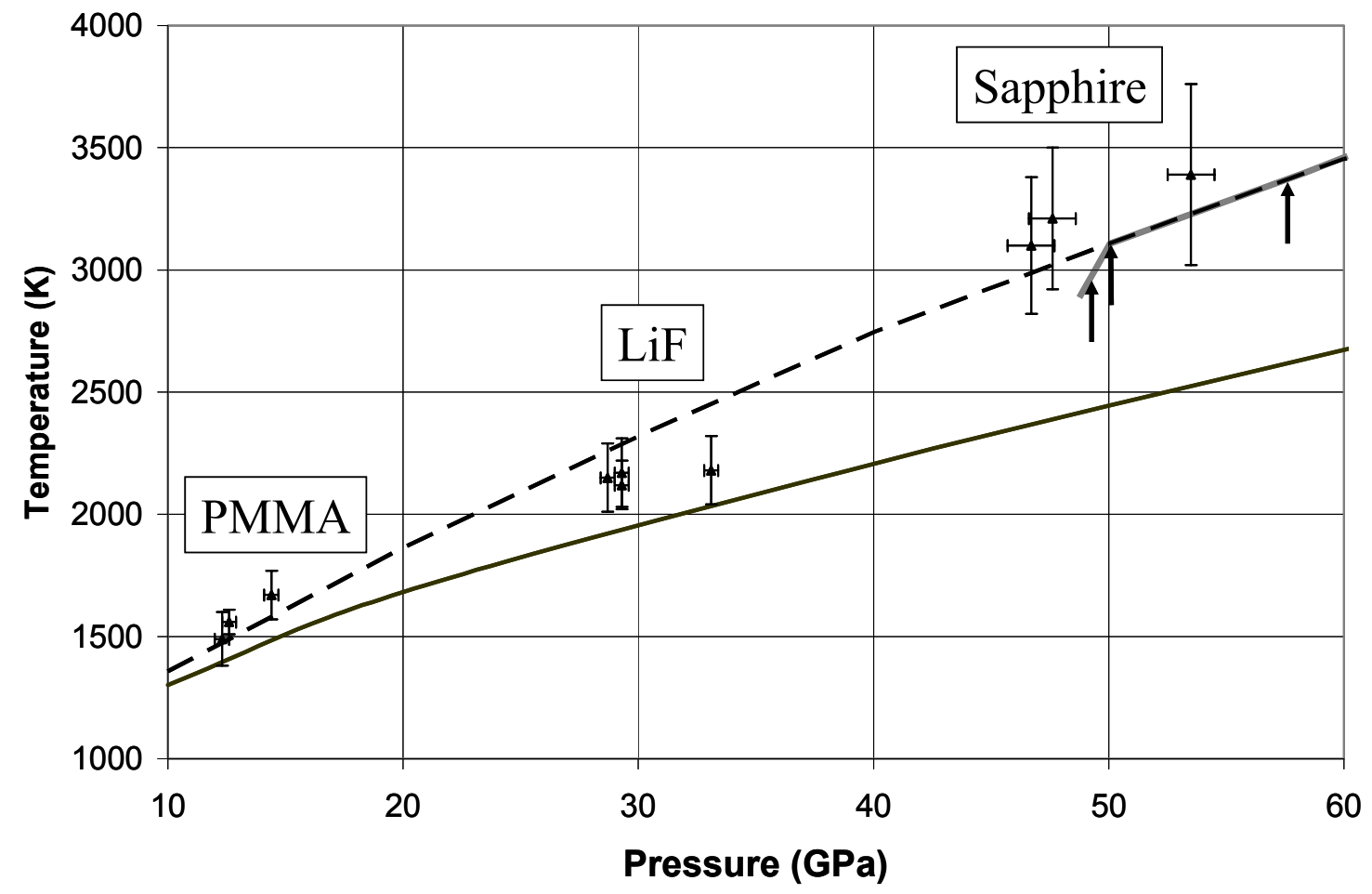

Fig. 18: Experimental data obtained on lead shocked at approximately 49, 50 and 57 GPa in pressure (solid triangles). These shock pressures were intentionally selected near the incipient melting point on the Hugoniot curve (about 50 GPa, cf. Ref. 4). We compare these data to the experimental melt curve obtained from diamond-anvil cell measurements (black solid line: data fit provided in Ref. 1) and a theoretical melt curve ${ }^{6}$ (black dotted line). A conceptual Hugoniot curve was drawn 
(grey line) in the vicinity of the theoretical melt curve. Vertical black arrows

indicate the three initial shock pressures in the lead samples.

\section{CONCLUSION}

We performed a set of experiments specifically designed to provide a measurement of the melt curve of lead up to approximately $54 \mathrm{GPa}$ in pressure (and up to about $3400 \mathrm{~K}$ in temperature). We also developed a new reflectometry diagnostic to obtain data on the reflectivity (and thus the emissivity) of the metallic samples under high-intensity shock loading. Not only does this diagnostic appear to be a possible way to detect phase transitions, but it also provides key data on the dynamic target emissivity to improve the accuracy of pyrometry measurements. The present shock results, in reasonable agreement with a previous theoretical investigation, show that the melting curve of lead is somewhat higher than suggested by static measurements at high pressure. The results on tin are also encouraging and will complement an on-going experimental study of the melt curve and the Hugoniot of this material.

\section{Acknowledgements}

We would like to thank W. A. Anderson, J. J. Bartos, R. R. Bartsch, B. A. Davis, D. O. Devore, F. P. Garcia, I. V. Garza, G. Gonzales, G. T. Gray, D. R Korzekwa, P. M. O'Gara, P. T. Reardon, C. C. Sparks and L. J. Tabaka (from Los Alamos National Laboratory and Bechtel Nevada) for their assistance with preparing the experiments. M. Vochosky (Bechtel Nevada) is thanked for her careful editing and helpful suggestions. This work was performed under the auspices of the U. S. Department of Energy at the 
University of California/Los Alamos National Laboratory (under contract No. W-7405ENG-36) and at Bechtel Nevada/Los Alamos Operations (under contract No. DE-AC0896NV11718). We gratefully acknowledge their support.

${ }^{1}$ B. K. Godwall, C. Meade, R. Jeanloz, A. Garcia, A. Y. Liu and M. L Cohen, Science 248, 462 (1990).

2 E. Y. Tonkov, High Pressure Phase Transformations, a Handbook, 635, Gordon and Breach Science Publishers, Philadelphia (1992).

3 J. F. Cannon, J. Phys. Chem. Ref. Data 3, 781 (1974).

${ }^{4}$ H. Bernier and P. Lalle, High Pressure in Research and Industry, Uppsala, 194 (1981), edited by C. M. Backman, T. Johannisson and L. Tegner, Arkitektkopia, Uppsala (1982).

${ }^{5}$ D. A. Boness, J. M. Brown and J. W. Shaner, Shock Waves in Condensed Matter, 115, (1987), edited by S. C. Schmidt and N. C. Holmes, Elsevier Science Publishers B. V. (1988).

6 J. L. Pélissier, Physica 126A, 271 (1984).

7 C. Mabire and P.L. Héreil, Shock Compression of Condensed Matter, Atlanta, 229, (2001), edited by M. D. Furnish, N. N. Thadhani and Y. Horie, American Institute of Physics, New York (2002).

8 D. Partouche-Sebban and J. L. Pélissier, Shock Waves 13, 69 (2003).

9 D. Partouche-Sebban, Shock Compression of Condensed Matter, Portland, 1293, (2003), edited by M. D. Furnish, Y. M. Gupta and J. W. Forbes, American Institute of Physics, New York (2004).

10 D. Partouche-Sebban, PhD dissertation, University of Paris XI, Orsay, 2002. 
11 R. G. McQueen and S. P. Marsh, J. Appl. Phys. 31, 1253 (1960).

12 P. A. Urtiew and R. J. Grover, J. Appl. Phys. 45, 140 (1974).

13 D. Partouche-Sebban, J. L. Pélissier, W. W. Anderson, R. S. Hixson and

D. B. Holtkamp, submitted for publication Physica B vol, pagenumber (2005).

14 C. Mabire and P. L. Héreil, Shock Compression of Condensed Matter, Atlanta, 1235, (2001), edited by M. D. Furnish, N. N. Thadhani and Y. Horie, American Institute of Physics, New York (2002).

15 W. J. Carter, S. P. Marsh, J. N. Fritz and R. G. McQueen, Accurate Characterization of the High-Pressure Environment, Gaithersburg, 147, (1968), edited by the National Bureau of Standards, Washington, DC (1971).

16 D. Partouche-Sebban, D. B. Holtkamp, P. Rodriguez, J. B. Stone, S. D. Borror, C. A. Kruschwitz and J. A. Young, submitted for publication R.S.I. vol, pagenumber (2005).

17 J. L. Wise and L. C. Chhabildas, Shock Waves in Condensed Matter, Spokane, 441, (1985), edited by Y. M. Gupta, Plenum Press, New York (1986).

18 J. L. Pélissier and D. Partouche-Sebban submitted for publication Physica B vol, pagenumber (2005).

19 D. Partouche-Sebban, J. L. Pélissier, W. W. Anderson, R. S. Hixson, D. B. Holtkamp, Shock Compression of Condensed Matter, Portland, 1289, (2003), edited by M. D. Furnish, Y. M. Gupta and J. W. Forbes, American Institute of Physics, New York (2004). 
20 S. P. Marsh, LASL Shock Hugoniot Data, University of California Press, Berkeley (1980).

21 J.L. Wise and L. C. Chhabildas, Shock Waves in Condensed Matter, Spokane, 441, (1985), edited by Y. M. Gupta, Plenum Press, New York (1986).

22 E. D. Olsen, Modern optical methods of analysis, McGraw-Hill book Inc., New York (1975).

${ }^{23}$ J. Hameury, Laboratoire National d'Essais, technical report no. E041533 (2004).

24 J. R. Asay, M. Shahinpoor, High-pressure shock compression of solids, SpringerVerlag, New York (1993).

25 Ya. B. Zeldovich and Yu. P. Raizer, Physics of shock waves and high-temperature hydrodynamic phenomena, Academic Press, New York (1967).

26 J. L. Pélissier et al., to be published.

27 N. Wetta and J. L. Pélissier, Physica A 289, 479 (2001).

DOE/NV11718--990. This work was supported by the U. S. Department of Energy National Nuclear Security Administration Nevada Operations Office, under Contract No. DE-AC0896NV11718.

Copyright Statement. By acceptance of this article, the publisher and/or recipient acknowledges the U. S. Government's right to retain a nonexclusive, royalty-free license in and to any copyright covering this paper. 
Disclaimer. This report was prepared as an account of work sponsored by an agency of the U. S. Government. Neither the U. S. Government nor any agency thereof, not any of their employees, nor any of their contractors, subcontractors or their employees, makes any warranty or representation, express or implied, or assumes any legal liability or responsibility for the accuracy, completeness, or usefulness of any information, apparatus, product, or process disclosed, or represents that its use would not infringe privately own rights. Reference herein to any specific commercial product, process, or service by trade name, trademark, manufacturer, or otherwise, does not necessarily constitute or imply its endorsement, recommendation, or favoring by the U. S. Government or any agency thereof. The views and opinions of authors expressed herein do not necessarily state or reflect those of the U. S. Government or any agency thereof. 